\title{
Introduzione alla conoscenza della sottofamiglia Aleocharinae della Guyana Francese: Parte I ' (Coleoptera, Staphylinidae)
}

\author{
Con 98 figure \\ ROBERTO PACE ${ }^{2}$ \\ ${ }^{1} 284^{\circ}$ "Contributo alla conoscenza delle Aleocharinae". \\ ${ }^{2}$ Via Vittorio Veneto, 13, 37032 Monteforte d’Alpone (Verona), Italia. - pace.ent@tiscali.it \\ Published on 2014-07-31
}

\section{Summary}

Description of 27 new species of the tribes Deinopsini, Pronomaeini, Hygronomiini, Oligotini, Gyrophaenini, Placusini: Myllena guyanensis n. sp., Amazonopora sulcicollis n. sp., Hygronoma guyanensis n. sp., Hygronoma struyvei n. sp., Hygronoma filiformis n. sp., Holobus guyanensis n. sp., Gyrophaena coraliensis n. sp., Gyrophaena amazonica n. sp., Gyrophaena comtensis n. sp., Gyrophaena perstriata n. sp., Gyrophaena ouaquiensis n. sp., Gyrophaena pilositheca n. sp., Gyrophaena guyabicornis n. sp., Gyrophaena perminima n.sp., Gyrophaena persubtilis n. sp., Gyrophaena guyasimplex n. sp., Gyrophaena struyvei n. sp., Gyrophaena schoelcheri n. sp., Gyrophaena microguyana n. sp., Gyrophaena guyanensis n. sp., Gyrophaena guytrina n. sp., Gyrophaena cayennensis n. sp., Gyrophaena, exiliphallus n. sp., Gyrophaena guyaspinosa n. sp., Brachida guyanensis n. sp., Brachychara struyvei n. sp., Placusa amazonica n. sp. Additional distributional records are reported for 15 species. Placusa graciensis is a new combination and new name for Amischa pumilio Bernhauer, 1927. Hygronoma calida (Bernhauer, 1908) is a new combination for Apimela calida Bernhauer, 1908. All new species are illustrated and compared with similar species. The holotype of Apimela calida BERNHAUER is illustrated for the first time.

\section{Key words}

Coleoptera, Staphylinidae, Aleocharinae, taxonomy, French Guiana.

\section{Zusammenfassung}

27 neue Arten der Tribus Deinopsini, Pronomaeini, Hygronomiini, Oligotini, Gyrophaenini, Placusini werden beschrieben: Myllena guyanensis n. sp., Amazonopora sulcicollis n. sp., Hygronoma guyanensis n. sp., Hygronoma struyvei n. sp., Hygronoma filiformis n. sp., Holobus guyanensis n. sp., Gyrophaena coraliensis n. sp., Gyrophaena amazonica n. sp., Gyrophaena comtensis n. sp., Gyrophaena perstriata n. sp., Gyrophaena ouaquiensis n. sp., Gyrophaena pilositheca n. sp., Gyrophaena guyabicornis n. sp., Gyrophaena perminima n. sp., Gyrophaena persubtilis n. sp., Gyrophaena guyasimplex n. sp., Gyrophaena struyvei n. sp., Gyrophaena schoelcheri n. sp., Gyrophaena microguyana n. sp., Gyrophaena guyanensis n. sp., Gyrophaena guytrina n. sp., Gyrophaena cayennensis n. sp., Gyrophaena, exiliphallus n. sp., Gyrophaena guyaspinosa n. sp., Brachida guyanensis n. sp., Brachychara struyvei n. sp., Placusa amazonica n. sp. Für 15 Arten werden zusätzliche Daten aufgeführt. Placusa graciensis ist eine neue Kombination und ein neuer Name für Amischa pumilio Bernhauer, 1927. Hygronoma calida (Bernhauer, 1908) ist eine neue Kombination für Apimela calida BERNHAUER, 1908. Jede neue Art wird illustriert und mit ähnlichen Arten verglichen. Der Holotypus von Apimela calida BERNHAUER wird zum ersten Mal illustriert. 


\section{Riassunto}

Descrizione di 27 nuove specie delle tribù Deinopsini, Pronomaeini, Hygronomiini, Oligotini, Gyrophaenini, Placusini: Myllena guyanensis n. sp., Amazonopora sulcicollis n. sp., Hygronoma guyanensis n. sp., Hygronoma struyvei n. sp., Hygronoma filiformis n. sp., Holobus guyanensis n. sp., Gyrophaena coraliensis n. sp., Gyrophaena amazonica n. sp., Gyrophaena comtensis n. sp., Gyrophaena perstriata n. sp., Gyrophaena ouaquiensis n. sp., Gyrophaena pilositheca n. sp., Gyrophaena guyabicornis n. sp., Gyrophaena perminima n. sp., Gyrophaena persubtilis n. sp., Gyrophaena guyasimplex n. sp., Gyrophaena struyvei n. sp., Gyrophaena schoelcheri n. sp., Gyrophaena microguyana n. sp., Gyrophaena guyanensis n. sp., Gyrophaena guytrina n. sp., Gyrophaena cayennensis n. sp., Gyrophaena, exiliphallus n. sp., Gyrophaena guyaspinosa n. sp., Brachida guyanensis n. sp., Brachychara struyvei n. sp., Placusa amazonica n. sp. Sono riportati dati distributivi supplementari per 15 specie. Placusa graciensis è nuova combinazione e nome nuovo per Amischa pumilio Bernhauer, 1927. Hygronoma calida (Bernhauer, 1908) è combinazione nuova per Apimela calida Bernhauer, 1908. Ogni specie nuova è illustrata e comparata con specie simili. L'holotypo di Apimela calida BernHAUER è illustrato per la prima volta.

\section{Introduzione}

La Guyana Francese è coperta per il $96 \%$ di foresta equatoriale che per lo più è una foresta primaria. La sua posizione vicino all'equatore e il suo affacciarsi sulloceano Atlantico le conferiscono una buona stabilità climatica. La temperatura annua media è di $26^{\circ} \mathrm{C}$. La grande stagione secca va da metà agosto a novembre. La Guyana Francese è una dalle regioni più umide al mondo con una variazione delle precipitazioni da $2000 \mathrm{~mm}$ a $4000 \mathrm{~mm}$ all'anno. Questi caratteri ambientali sono favorevoli allo sviluppo di una ricca fauna di Staphylinidae i cui rappresentanti prediligono i detriti umidi dell'ambiente forestale, un clima umido e piovoso. In caso di stagione secca molte specie della sottofamiglia Aleocharinae degli Staphylinidae si rifugiano o vivono in permanenza nei formicai, dove il clima è meno secco. Molte sono ancora le specie di Aleocharinae da scoprire nelle foreste equatoriali, rappresentate da esemplari piccoli o piccolissimi. Oggi si è generato il diffuso timore che molte di queste specie si estinguano prima ancora di venire scoperte e descritte a causa dell'impatto dell'uomo nel provocare estinzioni di massa. La scoperta e descrizione di nuove specie sono fondamentali per tentare di impedirne la sparizione. È questo il principale scopo del presente lavoro.

Alla città di Cayenna, capitale della Guyana Francese, fece tappa nel 1609 una spedizione promossa dal granduca Ferdinando I di Toscana e guidata dal capitano Thornton. La Guyana Francese fu in seguito colonizzata dai francesi. Nessuna spedizione scientifica con fini di raccogliere esemplari di fauna era aggregata a questi interventi europei. Perciò non sono stati nemmeno raccolti esemplari di Aleocharinae caratterizzati da taglia corporea minuta o minutissima e che quasi mai si possono incontrare senza l'uso di tecniche di ricerca specializzata come l'adozione del vaglio o di una rete di raccolta al volo con vettura o di trappole a caduta. Le successive raccolte hanno avuto carattere occasionale, come dimostrato dalla descrizione della prima specie della sottofamiglia Aleocharinae Aleochara cayen- nensis Castelnau (= Laporte), descritta nel 1835. Essa, come altre specie del genere, è attratta dal corpo umano sudato. Forse su esso la prima specie di Aleocharinae della Guyana Francese è stata raccolta, poi descritta. Castelnau non dà dati sulla sua raccolta. In seguito, le Aleocharinae, non proprio della Guyana Francese, ma di regioni vicine, come l'Amazzonia e il Brasile sono state oggetto di studio da parte di SHARP, $(1876,1883)$ e Bernhauer $(1908,1927)$ e, per specie mirmecofile e termitofile, da vari altri studiosi. Gran parte delle serie tipiche brasiliane, peruviane, cilene, ecuadoregne e antillane di Bernhauer, Fauvel, Fairmaire, Kraatz, e Erichson, sono state da me esaminate e dissezionate al fine di conoscere la forma dell'edeago e della spermateca, organi che offrono importanti e sicuri caratteri diagnostici al fine di riconoscimento delle specie e dei generi di appartenenza. È noto, infatti, che questi autori del passato quasi mai hanno esaminato e illustrato questi organi.

In questi ultimi anni le ricerche entomologiche in Sudamerica si sono intensificate, ma non in Guyana. Sono state oggetto di studio di miei lavori su materiali soprattutto dell'Ecuador, Perù, Venezuela, Paraguay e Cile. Le specie elencate e descritte nel presente lavoro e nelle successive parti di questa serie, fino alla sesta, sono le prime Aleocharinae massicciamente e accuratamente raccolte nella Guyana Francese. Di quelle eventualmente raccolte e "nascoste" nei vari musei non si hanno notizie. 


\section{Materiale e metodi}

Gli esemplari del presente lavoro sono stati raccolti molto di recente (anno 2011) in due settimane di novembre dal collega e naturalista belga Tim Struyve di Mechelen, che lavora anche in riserve naturali in Fiandra, Belgio. Egli ha raccolto Aleocharinae sia con retino su vettura in corsa, sia sotto nidi di formiche sia in loro colonne. Altre specie sono state da lui raccolte al margine di ruscelli, in frutta e nella lettiera, sempre in foresta. La tecnica di raccolta con retino su vettura mai è stata adottata in Guyana Francese. Si spiega così, con l'esplorazione di diversi biotopi, il gran numero di nuove specie raccolte: 131 su 167, 5 nuovi generi e 4 nuovi sottogeneri.

Anche lo studio tassonomico delle specie della sottofamiglia Aleocharinae della Guyana Francese, come quello d'altre regioni sudamericane, presenta seri problemi interpretativi che sono risolti in gran parte grazie all'esame della forma dell'organo copulatore maschile (edeago) e della spermateca. Gli esemplari sono stati dissezionati con lo scopo di includere le strutture genitali in balsamo del Canada (su piccoli rettangoli trasparenti di materiale di plastica, che accompagnano gli esemplari). Le strutture genitali sono state studiate con l'uso di un microscopio composto fino a 450 ingrandimenti e disegnate per mezzo di oculare a reticolo. Le misurazioni sono state eseguite mediante l'ausilio di un oculare con scala micrometrica. I caratteri dell'habitus sono stati osservati con microscopio stereoscopico fino a 100x. Tutti i disegni delle tavole e le foto sono stati da me eseguiti fino alla fase finale. Le tavole sono state da me composte al computer con Adobe Photoshop software.

\section{Acronimi}

Gli holotypi e paratypi delle nuove specie e altro materiale sono conservati nei seguenti istituti:

FMNHC: Field Museum of Natural History, Chicago IRSNB: Institut royal des Sciences naturelles de Belgique, Bruxelles

SDEI: Senckenberg Deutsches Entomologisches Institut, Müncheberg

\section{DEINOPSINI}

Adinopsis myllaenoides (KraAtz, 1857)

Deinopsis myllaenoides KraATZ, 1857: 38

Adinopsis myllaenoides, KLIMAszewski, 1979: 72

$10^{\star}$ e 3 우 ㅇ, Guyane Fr., Coralie, 15.XI.2011, leg. T. Struyve (IRSNB, SDEI).

Distribuzione: Sud U.S.A., Antille, Brasile, Perù.

Adinopsis ferruginea (SHARP, 1883)

Deinopsis ferruginea SHARP, 1883: 294

Adinopsis ferruginea, KLIMASzEwsKi, 1979: 75

7 esemplari, Guyane Fr., Coralie, 15.XI.2011, leg. T. Struyve (IRSNB, SDEI).

Distribuzione: Guatemala, Colombia.

\section{Adinopsis braziliensis KLIMASzEWSKI, 1979}

Adinopsis braziliensis Klimaszewski, 1979: 76

9 esemplari, Guyane Fr., Caussade, 7.XI.2011, leg. T. Struyve; 9 esemplari Guyane Fr., Tonate, 6.XI.2011, leg. T. Struyve; 1 o e 1 ㅇ, Guyane Fr., Saramaka, 13.XI.2011, leg. T. Struyve (IRSNB, SDEI).

Distribuzione: Brasile.

\section{Metadeinopsis longicornis (SHARP, 1876) \\ (Figg. 28-29)}

Deinopsis longicornis SHARP, 1876: 80

Metadeinopsis longicornis, KLIMASZEWSKI, 1979: 82

1 đ̊, Guyane Fr., Caussade, 7.XI.2011, leg. T. Struyve (SDEI).

Distribuzione: Brasile.

Nota: Finora questa specie era nota solo su esemplari femmina. Qui per la prima volta è pubblicato l'edeago (figg. 28-29). 


\section{PRONOMAEINI}

\section{Myllena guadalupensis PACE, 1987}

Myllena guadalupensis PACE, 1987: 187

5 esemplari, Guyane Fr., Caussade, 6-7.XI.2011, leg. T. Struyve; 1 ㅇ, Guyane Fr., Tonate, 6.XI.2011, leg. T. Struyve (IRSNB, SDEI).

Distribuzione: Brasile.

\section{Myllena guyanensis n. sp. (Figg. 1 e 30-32)}

Materiale tipico: Holotype $\sigma^{\star}$, Guyane Fr., Caussade, 7.XI.2011, leg. T. Struyve (IRSNB).

Paratypes: 9 es., stessa provenienza; 1 \% , Guyane Fr., Tonate, 6.XI.2011, leg. T. Struyve (IRSNB, SDEI).

Descrizione: Lunghezza 2,6 mm. Corpo lucido e giallo-bruno, capo bruno, margine posteriore degli uroterghi

\section{Amazonopora sulcicollis n. sp.}

(Figg. 2 e 33)

Materiale tipico: Holotype + , Guyane Fr., Coralie, 15.XI.2011, leg. T. Struyve (IRSNB).

Descrizione: Lunghezza 2,4 $\mathrm{mm}$. Corpo lucido e bruno-rossiccio, antenne e zampe rossicce. Occhi più corti della regione postoculare in visione dorsale. Secondo antennomero più lungo del primo, terzo lungo quanto il secondo, quarto a decimo trasversi. Reticolazione del capo forte, quella del pronoto molto forte, quella delle elitre evidente e quella dell'addome poligonale irregolare ben visibile. Punteggiatura di capo e pronoto invisibile. Granulosità delle elitre evidente e poco fitta, quella dell'addome saliente e più fitta sugli uroterghi liberi basali primo e secondo, rada sul terzo e quarto, molto rada sul quinto. Capo con debole solco mediano post-discale, pronoto con evidente solco mediano e con una stretta fossetta presso ciascun angolo posteriore. Spermateca fig. 33 .

Comparazioni: La nuova specie si distingue da quelle note per i caratteri dati nella seguente chiave:

1. Pronoto con solco mediano evidente. A. sulcicollis n. sp.

- Pronoto senza solco mediano

A. manausensis PACE, 1996; A. lescheni Ahn \& Ashe, 1999; A. brooksi Ahn \& Ashe, 1999

liberi rossiccio, pigidio giallo-rossiccio, antenne gialle con i due antennomeri basali giallo-rossicci, terzo a quinto bruni, zampe giallo-rossicce. Occhi più lunghi della regione postoculare in visione dorsale. Secondo antennomero molto più lungo del primo, terzo più corto del secondo, quarto a decimo più lunghi che larghi. Corpo interamente coperto di pubescenza sericea. Zampe con setola esterna molto corta, zampe posteriori con setola esterna ancor più corta. Edeago figg. 30-31, spermateca fig. 32.

Comparazioni: Per la forma della spermateca e per il margine posteriore degli uroterghi liberi, la nuova specie è simile a $M$. parvicollis KrAATZ, 1862 del Cile, di cui ho esaminato la serie tipica del sinonimo $M$. dilutipes FAIRMAIRE \& GERMAIN, 1861 (IRSNB). L'introflessione apicale del bulbo distale della nuova specie è lunga, quella di parvicollis breve. Il profilo apicale dell'edeago della nuova specie è arcuato, quello di parvicollis rettilineo. Le antenne della nuova specie sono gialle con i due antennomeri basali giallo-rossicci, terzo a quinto bruni, quelle di parvicollis sono brune con antennomero basale giallo.

Etimologia: Il nome della nuova specie deriva da quello della Guyana Francese.
Etimologia: La nuova specie prende nome dal solco mediano del suo pronoto.

Discussione: Ahn \& Ashe (1999) hanno osservato che il genere Amazonopora PACE, 1996 è simile ai generi della tribù Myllaenini, dimenticando che essa è sinonimo della tribù Pronomaeini. Perciò non esaminano i caratteri del genere Pronomaea ErICHSON, 1837. Avrebbero potuto cosi verificare che tra Amazonopora e Pronomaea esistono strette affinità. Amazonopora è il genere sudamericano vicariante del paleartico Pronomaea e dell'africano Nopromaea CAmeron, 1930. 


\section{HYGRONOMIINI}

\section{Hygronoma calida (BeRNHAUER, 1908) comb. n.}

Apimela calida Bernhauer, 1908: 355

Materiale esaminato: Holotype $0^{\star}$, Brazil, S. Paulo, Estac. Raiz da Serra, Dr. Ihering, Apimela calida Brnh. (FMNHC).

Paratypes: $10^{\Uparrow}$ e $1 \%$, stessa provenienza (FMNHC).

Nota: Il mio esame della serie tipica di Apimela calida BERnHAUER, mi ha permesso di osservare che la formula tarsale non è 5-5-5 come in Apimela Mulsant \& Rey, 1874 della tribù Oxypodini, ma 4-4-4 della tribù Hygronomiini. La ligula in A. calida, inoltre, è intera e non divisa in due larghi lobi come in Apimela. Per questi caratteri calida va attribuita a Hygronoma ERICHSON, 1837. In una futura revisione tassonomica delle Aleocharinae dovrà essere riconosciuto che Hygronoma è molto affine al genere Apimela Mulsant \& ReY, 1874 a causa della forma della spermateca, nonostante la differente formula tarsale. È spiegabile così anche l'errore di attribuzione generica di BERnHAUER.

Questa specie si distingue dalle nuove qui descritte per la chiave seguente: dorsale. Secondo antennomero lungo quanto il primo, terzo più corto del secondo, quarto a decimo fortemente trasversi. Reticolazione di capo ed elitre evidente, quella di pronoto e addome molto superficiale. Punteggiatura del capo fitta, fine e molto evanescente. Granulosità di pronoto, elitre e addome fitta, fine e superficiale, sugli uroterghi liberi quarto e quinto sparsa. Edeago figg. 34-35, spermateca fig. 36.

Etimologia: Il nome della nuova specie deriva da quello della Guyana Francese.

\section{Hygronoma struyvei n. sp.}

(Figg. 4 e 37-39)

Materiale tipico: Holotype $\sigma^{\star}$, Guyane Fr., Caussade, 6.XI.2011, leg. T. Struyve (IRSNB).

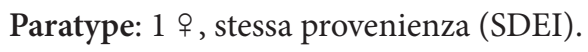

Descrizione: Lunghezza $1,8 \mathrm{~mm}$. Corpo lucido e bruno-rossiccio, capo bruno, antenne nere con i due antennomeri basali giallo-rossicci, zampe gialle. Occhi più corti della regione postoculare in visione dorsale. Secondo antennomero più corto del primo, terzo più corto del secondo, quarto a decimo fortemente trasversi. Reticolazione del capo e degli uroterghi liberi quarto e

1. Base dell'addome giallo-bruna o giallo-rossiccia 2

- Base dell'addome bruna o bruno-rossiccia

2. Pronoto rossiccio, base dell'addome giallo-rossiccia; reticolazione del capo assente, reticolazione dell'addome evidente, sul quinto urotergo libero forte; protuberanze ventrali dell'edeago strette e molto distanziate, in visione ventrale. Lunghezza $2 \mathrm{~mm}$. Brasile H. calida (BERNHAUER)

- Pronoto e base dell'addome giallo-bruni; reticolazione del capo evidente, quella dell'addome molto superficiale; protuberanze ventrali dell'edeago larghe e contigue, in visione ventrale. Lunghezza 1,3 mm. Guyana Francese .....

H. guyanensis n. sp.

3. Occhi più sporgenti, elitre corte, pronoto bruno-rossiccio, pigidio bruno-rossiccio; spermateca maggiore, con bulbo distale corto. Lunghezza 1,8 mm. Guyana Francese H. struyvei n. sp.

- Occhi meno sporgenti, elitre lunghe, pigidio giallo-rossiccio; spermateca minore con bulbo distale lungo. Lunghezza $1,8 \mathrm{~mm}$. Guyana Francese H. filiformis n. sp.

\section{Hygronoma guyanensis n. sp}

(Figg. 3 e 34-36)

Materiale tipico: Holotype $\sigma^{\star}$, Guyane Fr., Caussade, 7.XI.2011, leg. T. Struyve (SDEI).

Paratypes: 12 esemplari, stessa provenienza (IRSNB, SDEI).

Descrizione: Lunghezza 1,3 mm. Corpo lucido e giallo-bruno, capo e uroterghi liberi quarto e quinto bruni, antenne brune con i tre antennomeri basali gialli, zampe gialle. Occhi più corti della regione postoculare in visione quinto evidente, quella del pronoto non visibile, quella delle elitre e dei tre uroterghi liberi basali superficiale. Granulosità del corpo fine, fitta e saliente, sul quinto urotergo libero meno fitta. Fronte impressa. Edeago figg. 37-38, spermateca fig. 39.

Etimologia: La nuova specie è dedicata al suo raccoglitore Tim Struyve, entomologo e consulente di pianificazione di Mechelen, Belgio. 


\section{Hygronoma filiformis n. sp.}

(Figg. 5 e 40)

Materiale tipico: Holotype + , Guyane Fr., Barrage Petit Saut, 10.XI.2011, leg. T. Struyve (IRSNB).

Paratype: 1 , stessa provenienza (SDEI).

Descrizione: Lunghezza $1,8 \mathrm{~mm}$. Corpo lucido e bruno, pronoto giallo-bruno, pigidio giallo-rossiccio, antenne brune con i tre antennomeri basali giallo-bruni. Occhi più corti della regione postoculare in visione dorsale. Secondo antennomero più corto del primo, terzo più corto del secondo, quarto a decimo fortemente trasversi. Reticolazione del capo forte, quella di pronoto e addome superficiale, quella delle elitre evidente. Granulosità di capo ed elitre poco distinta, quella del pronoto quasi invisibile, quella dell'addome fitta, ma sul quinto urotergo libero sparsa. Spermateca fig. 40.

Etimologia: La nuova specie prende nome dalla forma del corpo stretta e lunga come un filo.

\section{OLIGOTINI}

Oligota parva KRAATZ, 1862

Oligota parva KRAATZ, 1862 300; FrAnK, 1972: 140

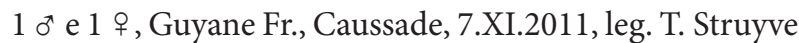
(IRSNB, SDEI).

Distribuzione: Europa, Antille, Messico, Colombia, Argentina, Australia.

\section{Holobus chrysopygus (KRAATZ, 1859)}

Oligota chrysopyga KraAtz, 1859: 45; Frank, 1972: 131

Holobus chrysopygus, Smetana, 2004: 453

19 esemplari, Guyane Fr., Caussade, 7.XI.2011, leg. T. Struyve (IRSNB, SDEI).

Distribuzione: Specie cosmopolita.

\section{Holobus testaceorufus BerNHAUER, 1923}

Oligota testaceorufa Bernhauer, 1923: 144; FrANK, 1972: 135

1 ơ $^{\star}$ Guyane Fr., Caussade, 7.XI.2011, leg. T. Struyve (IRSNB).

Distribuzione: Antille.
Nota: Le elitre degli esemplari della Guyana Francese sono nere e non giallo-rossicce come negli esemplari delle Antille, ma il profilo ventrale dell'edeago è identico negli esemplari delle due aree geografiche.

\section{Holobus hypocyptinus BeRnHAUER, 1923}

Oligota hypocyptina BernhaUer, 1923: 143; Frank, 1972: 134

3 esemplari, Guyane Fr., Caussade, 7.XI.2011, leg. T. Struyve (SDEI).

Distribuzione: Guadeloupe.

\section{Holobus guyanensis n. sp.}

(Figg. 6 e 41-42)

Materiale tipico: Holotype $\sigma^{*}$, Guyane Fr., Caussade, 7.XI.2011, leg. T. Struyve (IRSNB).

Paratypes: 4 esemplari, stessa provenienza (IRSNB, SDEI).

Descrizione: Lunghezza $0,9 \mathrm{~mm}$. Corpo lucido e molto convesso, nero-bruno, margini laterale e posteriore del pronoto rossicci, pigidio giallo-rossiccio. Occhi più lunghi della regione postoculare in visione dorsale. Secondo antennomero più lungo del primo, terzo più corto del secondo, quarto a nono più lunghi che larghi. Reticolazione di capo e pronoto non visibile, quella di elitre e addome molto superficiale. Granulosità di capo e pronoto molto fine e molto evanescente, quella delle elitre quasi invisibile, quella dell'addome molto saliente. Edeago fig. 41, spermateca fig. 42.

Comparazioni: Ledeago della nuova specie è simile a quello di H. bruchi Bernhauer, 1927 del Brasile, di cui ho esaminato un maschio di S. Paolo (Hungarian Natural History Museum, Budapest). Ledeago della nuova specie, pur avendo una gibbosità presso la "crista apicalis" come in bruchi, è di lunghezza è nettamente inferiore $(0,18 \mathrm{~mm}$, mentre in bruchi è di $0,31 \mathrm{~mm})$. Il lungo flagello interno dell'edeago di bruchi manca nell'edeago della nuova specie. La pelosità interna dell'edeago della nuova specie manca in bruchi. La clava antennale di bruchi è dilatata ed evidente, quella della nuova specie stretta e poco appariscente.

Etimologia: Il nome della nuova specie deriva da quello della Guyana Francese. 


\section{GYROPHAENINI}

\section{Gyrophaena (Phanerota) vecta PACE, 1990}

Gyrophaena (Phanerota) vecta PACE, 1990: 55

15 es., Guyane Fr., Caussade, 7.XI.2011, leg. T. Struyve (IRSNB, SDEI).

Distribuzione: Argentina, Perù.

\section{Gyrophaena (Phanerota) perinsidiosa PACE, 2008}

Gyrophaena (Phanerota) perinsidiosa PACE, 2008: 238

1 o $^{*}$ e 5 우 우, Guyane Fr., Caussade, 7.XI.2011, leg. T. Struyve (IRSNB, SDEI).

Distribuzione: Perù.

Gyrophaena (Phanerota) coraliensis n. sp.

(Figg. 7 e 43-45)

Materiale tipico: Holotype $\sigma^{*}$, Guyane Fr., Coralie, 15.XI.2011, leg. T. Struyve (IRSNB).

Paratypes: 4 우 우, stessa provenienza; 3 우 우, Guyane Fr. Saramaka, 13.XI.2011, leg. T. Struyve (IRSNB, SDEI).

Descrizione: Lunghezza 1,9 mm. Corpo lucido e giallo-rossiccio, capo bruno-rossiccio, antenne giallo-rossicce con i tre antennomeri basali gialli, zampe giallo-rossicce. Occhi enormi, in visione dorsale. Secondo antennomero più corto del primo, terzo più corto del secondo, quarto $\mathrm{e}$ quinto lunghi quanto larghi, sesto a decimo debolmente trasversi. Reticolazione del capo molto superficiale, quella del pronoto molto trasversa, ondulata e molto evanescente, quella delle elitre molto trasversa, obliqua e forte, quella dell'addome poligonale irregolare fine $\mathrm{e}$ evanescente, ma sul quinto urotergo libero evidente. Punteggiatura del capo rada ed evanescente, quella del pronoto sparsa e forte, quella delle elitre doppia, fine e rada, forte e assai sparsa.Edeago fig. 43, spermateca fig. 44, sesto urotergo libero del maschio fig. 45.

Comparazioni: La nuova specie per la forma dell'edeago è simile a $G$. fasciata SAY, 1834 del N America, ma il pezzo copulatore interno dell'edeago descrive un ampio arco nella nuova specie e non uno stretto arco con sinuosità apicali come in fasciata. La concavità ventrale dell'edeago della nuova specie è triangolare, quella di fasciata è ampiamente arcuata. I due lobi laterali del sesto urotergo libero del maschio di fasciata sono trasformati in due spine nella nuova specie. Il pronoto di fasciata non è reticolato, quello della nuova specie lo è evidentemente, anche se superficialmente.
Etimologia: Il nome della nuova specie deriva da quello della sua località di raccolta, Coralie.

Nota: L’edeago della nuova specie era già estratto e incollato sul cartellino porta insetti. La probabile amputazione dell'apice dell'edeago perciò non è stata causata dallo scrivente, ma dal collega che l'ha estratto e ha rinunciato allo studio.

\section{Gyrophaena (Phanerota) amazonica n. sp. \\ (Figg. 8 e 46-48)}

Materiale tipico: Holotype $\sigma^{\star}$, Guyane Fr., Barrage Petit Saut, 10.XI.2011, leg. T. Struyve (SDEI).

Paratypes: 2 우, stessa provenienza (IRSNB).

Descrizione: Lunghezza 2,4 mm. Corpo lucido. Capo bruno, pronoto ed elitre giallo-bruni, addome giallo-rossiccio con quarto urotergo libero rossiccio, antenne e zampe gialle. Occhi enormi, in visione dorsale. Secondo antennomero più corto del primo, terzo più corto del secondo, quarto a decimo debolmente trasversi. Reticolazione del capo non visibile, quella del pronoto molto trasversa e molto superficiale, quella delle elitre molto trasversa evidente, quella dell'addome evanescente. Punteggiatura di capo ed elitre sparsa e superficiale, quella del pronoto sparsa evidente, quella dell'addome debolmente fitta. Edeago fig. 46, spermateca fig. 47, sesto urotergo libero del maschio fig. 48.

Comparazioni: Il dente preapicale ventrale dell'edeago della nuova specie è simile a quello dell'edeago di G. quassa SHARP, 1876 dell'Amazzonia. La parte apicale ventrale dell'edeago della nuova specie è lunga e stretta, in visione laterale, quella di quassa è molto corta. L'armatura genitale interna dell'edeago della nuova specie è molto larga nella regione preapicale, stretta in quassa.

Etimologia: Il nome della nuova specie deriva da quello dell'Amazzonia.

\section{Gyrophaena (Gyrophaena) comtensis n. sp.} (Figg. 9 e 49-51)

Materiale tipico: Holotype $\sigma^{\star}$, Guyane Fr., Coralie, 15.XI.2011, leg. T. Struyve (IRSNB).

Paratypes: $2 o^{\star} \sigma^{\star}$ e 1 ㅇ stessa provenienza (IRSNB, SDEI).

Descrizione: Lunghezza 1,6 mm. Corpo lucido e giallo-rossiccio, antenne brune con i tre antennomeri basali gialli, zampe giallo-rossicce. Occhi poco più lunghi della regione postoculare, in visione dorsale. Secondo antennomero più corto del primo, terzo più corto del secondo, quarto a decimo trasversi. 
Reticolazione del capo molto superficiale, quella di pronoto e addome evidente, quella delle elitre trasversa e ben visibile. Punteggiatura del capo forte, non fitta e assente sulla fascia longitudinale mediana, quella del pronoto poco profonda. Granulosità delle elitre molto evanescente, quella dell'addome invisibile. Primo urotergo libero del maschio con paratergiti espansi a triangolo all'esterno. Edeago fig. 49, spermateca fig. 50, sesto urotergo libero del maschio fig. 51.

Comparazioni: Il lobo mediano del sesto urotergo libero del maschio della nuova specie è presente anche in G. cornelli PACE, 2008 del Perù, ma le spine laterali a questo lobo sono molto corte nella nuova specie, lunghe in cornelli. La granulosità delle elitre di cornelli è allineata in due file oblique, nella nuova specie è sparsa e molto superficiale.

Etimologia: La nuova specie deriva il suo nome dal fiume Comtè della Guyana Francese.

\section{Gyrophaena (Gyrophaena) perstriata n. sp. (Figg. 10 e 52-53)}

Materiale tipico: Holotype + , Guyane Fr., Coralie, 15.XI.2011, leg. T. Struyve (IRSNB).

Descrizione: Lunghezza 2,4 $\mathrm{mm}$. Corpo lucido e bruno-rossiccio, base dell'addome e pigidio giallo-rossicci, antenne giallo-rossicce con i tre antennomeri basali e l'undicesimo gialli, zampe gialle. Occhi più corti della regione postoculare, in visione dorsale. Secondo antennomero più corto del primo, terzo più corto del secondo, quarto trasverso, quinto a decimo lunghi quanto larghi. Reticolazione di capo e addome evidente, quella di pronoto ed elitre molto superficiale. Punteggiatura del capo assente sul disco, forte e fitta nella regione occipitale, quella del pronoto forte e sparsa, quella delle elitre ben visibile. Granulosità dell'addome finissima ed evanescente con alcuni granuli salienti. Quinto urotergo libero della femmina con quattro deboli carene obliquamente convergenti verso la linea mediana. Spermateca fig. 52, sesto urotergo libero della femmina fig. 53.

Comparazioni: Per la presenza di occhi poco sviluppati, per le carene oblique sul quinto urotergo libero della femmina e per la forma della spermateca, la nuova specie è simile a G. spatulata PACE, 1996 dell'Ecuador. Se ne distingue per la spermateca con bulbo distale maggiore di quello di spatulata, largo $0,054 \mathrm{~mm}$, mentre quello della nuova specie è largo $0,04 \mathrm{~mm}$. L'undicesimo antennomero è giallo nella nuova specie, bruno in spatulata.

Etimologia: La nuova specie ha nome che significa "molto striata" per la presenza di estese strie interne della spermateca.

\section{Gyrophaena (Gyrophaena) ouaquiensis n. sp.}

(Figg. 11 e 54-55)

Materiale tipico: Holotype $\sigma^{\star}$, Guyane Fr., Saramaka, 13.XI.2011, leg. T. Struyve (SDEI).

Paratype: $1 \overbrace{}^{\star}$, stessa provenienza (IRSNB).

Descrizione: Lunghezza 2,1 mm. Corpo lucido. Avancorpo bruno, addome giallo-rossiccio con uroterghi liberi quinto e sesto bruni, antenne e zampe giallo-rossicce. Occhi lunghi quanto la regione postoculare, in visione dorsale. Secondo antennomero più corto del primo, terzo più corto del secondo, quarto trasverso, quinto a decimo lunghi quanto larghi. Reticolazione del capo, tranne che sul disco, ed elitre superficiale, quella del pronoto molto evanescente, quella del disco del capo e dell'addome evidente. Punteggiatura del capo ben visibile e sparsa, quella del pronoto molto sparsa e forte. Granulosità delle elitre fine e saliente, tra essa si trovi una fine punteggiatura. Granulosità dell'addome fine. Quinto urotergo libero del maschio con due fini carene convergenti all'indietro a ciascun lato della linea mediana. Edeago fig. 54, sesto urotergo libero del maschio fig. 55.

Comparazioni: Per la forma dell'edeago la nuova specie è simile a G. lobata CASEY, 1906 del N America. Se ne distingue per il pronoto bruno, mentre è giallo-rossiccio con disco oscurato in lobata, per il pronoto nettamente punteggiato, mentre in lobata la punteggiatura è quasi invisibile. Il profilo ventrale dell'edeago della nuova specie è arcuato, quello di lobata è sinuoso. Lapice dell'edeago della nuova specie è arrotondato, senza appendice apicale, quello dell'edeago di lobata ha una corta appendice.

Etimologia: La nuova specie deriva il suo nome dal fiume Ouaqui della Guyana Francese.

\section{Gyrophaena (Gyrophaena) pilositheca n. sp. (Figg. 12 e 56-57)}

Materiale tipico: Holotype + , Guyane Fr., Coralie, 15.XI.2011, leg. T. Struyve (IRSNB).

Descrizione: Lunghezza 2,3 mm. Corpo lucido e rossiccio, addome giallo-rossiccio, antenne brune con gli antennomeri basali secondo e terzo giallo-rossicci, il primo bruno, zampe giallo-rossicce. Occhi lunghi quanto la regione postoculare, in visione dorsale. Secondo antennomero più corto del primo, terzo più corto del secondo, quarto trasverso, quinto e sesto lunghi quanto larghi, settimo a decimo debolmente trasversi. Reticolazione del capo forte, quella del pronoto evidente, quella di elitre e addome poligonale irregolare ben visibile. Punteggiatura del capo composta di punti fini e superficiali e due forti punti, ciascuno ai lati del disco. Punteggiatura 
del pronoto con punti evidenti sparsi, alcuni forti, altri superficiali e fini. Granulosità di elitre e addome fine e molto evanescente. Quinto urotergo libero della femmina con una spina mediana acuta, lunga e saliente all'indietro. Spermateca fig. 56, sesto urotergo libero della femmina fig. 57.

Comparazioni: Per la forma del sesto urotergo libero della femmina la nuova specie potrebbe essere tassonomicamente vicina a $G$. comtensis n. sp. sopra descritta. Se ne distingue per l'assenza di spine laterali al margine posteriore del sesto urotergo libero della femmina, presenti in comtensis. La spermateca della nuova specie ha una parte pelosa, assente in comtensis, sostituita da spire.

Etimologia: La nuova specie ha nome che significa "spermateca pelosa".

\section{Gyrophaena (Eumicrota) atoma CASEY, 1906}

Eumicrota atoma CASEY, 1906: 284

Gyrophaena (Eumicrota) atoma; SEEvers, 1951: 738

8 esemplari, Guyane Fr., Caussade, 7.XI.2011, leg. T. Struyve; 10 , Guyane Fr., Barrage Petit Saut, 10.XI.2011, leg. T. Struyve (IRSNB, SDEI).

Distribuzione: U.S.A.

\section{Gyrophaena (Eumicrota) veninfida PACE, 2011}

Gyrophaena veninfida PACE, 2011: 46

$20^{\top} o^{\star}$ e 4 우 우, Guyane Fr., Caussade, 7.XI.2011, leg. T. Struyve; 1 ㅇ, Guyane Fr., Coralie, 15.XI.2011, leg. T. Struyve (IRSNB, SDEI).

Distribuzione: Venezuela.

\section{Gyrophaena (Eumicrota) guyabicornis n. sp.} (Figg. 13 e 58-59)

Materiale tipico: Holotype $\sigma^{\star}$, Guyane Fr., Caussade, 7.XI.2011, leg. T. Struyve (IRSNB).

Descrizione: Lunghezza 1,2 mm. Corpo lucido e bruno, base dell'addome giallo-bruna, antenne giallo-brune con i tre antennomeri basali gialli, zampe gialle. Occhi più lunghi della regione postoculare, in visione dorsale. Secondo antennomero più corto del primo, terzo più corto del secondo, quarto a decimo trasversi. Reticolazione di capo e pronoto superficiale, quella di elitre e addome evidente. Punteggiatura del capo molto evanescente. Granulosità del pronoto poco fitta, quella delle elitre saliente. Due pliche oblique presso ciascun angolo posteriore interno delle elitre del maschio. Sesto urotergo libero del maschio con due cornetti mediani salienti (fig. 59). Edeago fig. 58, sesto urotergo libero del maschio fig. 59.

Comparazioni: La parte apicale ventrale dell'edeago della nuova specie è uguale a quella dell'edeago di G. ferrariae PACE, 1991, dell'isola di S. Lucia (Antille). La spina apicale dorsale dell'edeago della nuova specie manca in ferrariae e il margine posteriore del sesto urotergo libero del maschio della nuova specie è lineare fig. 59 , mentre è diviso in due spine laterali e un lobo mediano in ferrariae.

Etimologia: Il nome della nuova specie significa "due corna della Guyana Francese". Le corna sono quelle del sesto urotergo libero del maschio.

\section{Gyrophaena (Eumicrota) perminima n. sp. (Figg. 14 e 60-63)}

Materiale tipico: Holotype $\sigma^{\star}$, Guyane Fr., Coralie, 15.XI.2011, leg. T. Struyve (SDEI).

Paratypes: 5 esemplari, stessa provenienza (IRSNB, SDEI).

Descrizione: Lunghezza 1,2 mm. Corpo lucido e bruno, addome giallo-rossiccio con uroterghi liberi quarto e quinto bruni, antenne giallo-brune con i tre antennomeri basali gialli, zampe gialle. Occhi più lunghi della regione postoculare, in visione dorsale. Secondo antennomero lungo quanto il primo, terzo più corto del secondo, quarto a decimo trasversi. Reticolazione dell'avancorpo evidente, quella dell'addome invisibile. Granulosità del capo poco visibile, quella di pronoto ed elitre fitta, fine e saliente. Elitre con tre file di granuli allineati a formare tre carene oblique presso ciascun angolo posteriore interno. Quinto urotergo libero del maschio con due brevi carene mediane affilate e strette, tra loro ben distanziate. Edeago fig. 60, spermateca fig. 61, sesto urotergo libero del maschio fig. 62, sesto urotergo libero della femmina fig. 63

Comparazioni: L'edeago della nuova specie ha parte apicale ventrale quasi rettilinea come in quello di G. golbachi PACE, 1990 dell'Argentina, ma la lunghezza dell'edeago della nuova specie è $0,1 \mathrm{~mm}$, quella di golbachi $0,19 \mathrm{~mm}$. Il sesto urotergo libero del maschio della nuova specie ha un solo lobo mediano al margine posteriore, quello di golbachi due.

Etimologia: La nuova specie ha nome che significa "estremamente piccola" in riferimento alla sua ridottissima lunghezza del corpo. 


\section{Gyrophaena (Eumicrota) persubtilis n. sp.}

(Figg. 15 e 64-65)

Materiale tipico: Holotype $\sigma^{\star}$, Guyane Fr., Coralie, 15.XI.2011, leg. T. Struyve (IRSNB).

Descrizione: Lunghezza $0,8 \mathrm{~mm}$. Corpo lucido e rossiccio, addome giallo-rossiccio con urotergo libero rossiccio, antenne giallo-brune con i tre antennomeri basali gialli, zampe gialle. Occhi più lunghi della regione postoculare, in visione dorsale. Secondo antennomero lungo quanto il primo, terzo più corto del secondo, quarto a decimo trasversi. Reticolazione di capo e pronoto invisibile, quella di elitre e addome superficiale. Punteggiatura del capo evanescente, quella del pronoto poco visibile. Granulosità delle elitre superficiale, quella dell'addome poco distinta. Edeago fig. 64, sesto urotergo libero del maschio fig. 65 .

Comparazioni: L’edeago della nuova specie è simile a quello di G. ferrariae PACE, 1991 dell'isola di S. Lucia (Antille). Se ne distingue per la parte apicale ventrale più sottile, con apice non rivolto al lato dorsale come in ferrariae. Il sesto urotergo libero del maschio della nuova specie ha un lobo mediano triangolare al margine posteriore, quello di ferrariae ha un largo lobo mediano tra due lunghe spine laterali.

Etimologia: La nuova specie ha nome che significa "molto sottile" con riferimento alla sottigliezza della lama sternale delledeago.

\section{Gyrophaena (Eumicrota) guyasimplex n. sp. (Figg. 16 e 66-68)}

Materiale tipico: Holotype ơ, Guyane Fr., Caussade, 7.XI.2011, leg. T. Struyve (IRSNB).

Paratypes: 7 우, stessa provenienza (IRSNB, SDEI).

Descrizione: Lunghezza 1,2 $\mathrm{mm}$. Corpo lucido e bruno-rossiccio, uroterghi liberi quarto e quinto bruni, antenne rossicce con i tre antennomeri basali gialli, zampe gialle. Occhi poco più lunghi della regione postoculare, in visione dorsale. Secondo antennomero più corto del primo, terzo più corto del secondo, quarto a decimo trasversi. Reticolazione del capo superficiale, quella di pronoto, elitre e addome evidente, sul quinto urotergo libero del maschio forte. Punteggiatura del capo poco distinta. Granulosità di pronoto ed elitre fitta e saliente. Quinto urotergo libero del maschio con due callosità mediane salienti. Edeago fig. 66, spermateca fig. 67 , sesto urotergo libero del maschio fig. 68.

Comparazioni: L'edeago della nuova specie è simile a quello di G. golbachi PACE, 1990 dell'Argentina. Se ne distingue per l'armatura genitale interna semplice e non avvolta in numerose spire come in golbachi. Il sesto urotergo libero del maschio è molto differente nelle due specie: due spine laterali molto distanziate al margine posteriore nella nuova specie, due lobi triangolari mediani contigui in golbachi.

Etimologia: La nuova specie ha nome che significa "semplice della Guyana Francese" a motivo della semplicità della forma dell'edeago, rispetto a quello più complesso di golbachi.

\section{Gyrophaena (Eumicrota) struyvei n. sp. \\ (Figg. 17 e 69-71)}

Materiale tipico: Holotype $\sigma^{*}$, Guyane Fr., Caussade, 7.XI.2011, leg. T. Struyve (IRSNB).

Paratypes: 15 esemplari, stessa provenienza; $4 \overbrace{}^{\star} \sigma^{\star}$, Guyane Fr., Saramaka, 13.XI.2011, leg. T. Struyve (IRSNB, SDEI).

Descrizione: Lunghezza 1,2 mm. Corpo lucido e bruno, uroterghi liberi quarto e quinto neri, antenne bruno-rossicce con i tre antennomeri basali gialli, zampe gialle. Occhi più lunghi della regione postoculare, in visione dorsale. Secondo antennomero più corto del primo, terzo più corto del secondo, quarto a decimo trasversi. Reticolazione di capo e ed elitre superficiale, quella del pronoto molto evanescente, quella dell'addome evidente. Punteggiatura del capo forte e assente sulla fascia longitudinale mediana, quella del pronoto composta di alcuni punti più impressi e altri superficiali. Granulosità delle elitre molto sparsa e superficiale, quella dell'addome saliente solo lungo il margine posteriore di ciascun urotergo libero. Edeago fig. 69, sesto urotergo libero del maschio figg. 70-71.

Comparazioni: L'edeago della nuova specie è simile a quello di G. socia ErICHSON, 1840 del N America, ma è privo della lunga appendice apicale dorsale, presente in socia. Il sesto urotergo libero del maschio della nuova specie è bisinuato al margine posteriore, quello di socia ha due lobi triangolari.

Etimologia: La nuova specie è dedicata al suo raccoglitore Tim Struyve, entomologo e consulente di pianificazione di Mechelen, Belgio.

Gyrophaena (Eumicrota) schoelcheri n. sp.

(Figg. 18 e 72-74)

Materiale tipico: Holotype $0^{\star}$, Guyane Fr., Caussade, 7.XI.2011, leg. T. Struyve (SDEI).

Paratype: 1 ㅇ, stessa provenienza (IRSNB).

Descrizione: Lunghezza $1 \mathrm{~mm}$. Corpo lucido e bruno-rossiccio, base dell'addome giallo-rossiccia, antenne 
giallo-brune con i due antennomeri basali e base del terzo gialli, zampe gialle. Occhi più lunghi della regione postoculare, in visione dorsale. Secondo antennomero più corto del primo, terzo più corto del secondo, quarto a decimo trasversi. Reticolazione del capo superficiale, quella del pronoto evidente, quella dell'addome ben visibile. Punteggiatura di capo e pronoto fitta e molto evanescente. Granulosità dell'addome solo lungo il margine posteriore degli uroterghi liberi. Quinto urotergo libero del maschio con due tubercoli mediani molto salienti. Edeago fig. 72, spermateca fig. 73, sesto urotergo libero del maschio fig. 74 .

Comparazioni: I due lobi del margine posteriore del sesto urotergo libero del maschio della nuova specie sono presenti anche in G. canaimensis PACE, 2011 del Venezuela, ma quelli della nuova specie sono corti. La parte apicale dell'edeago della nuova specie è sinuata, in visione laterale, quella di canaimensis è arcuata.

Etimologia: La nuova specie è dedicata a Victor Schoelcher, presidente della commissione che decretò l'abolizione della schiavitù nel 1848 nella Guyana Francese.

\section{Gyrophaena (Eumicrota) microguyana n. sp.}

(Figg. 19 e 75-77)

Materiale tipico: Holotype $\sigma^{\star}$, Guyane Fr., Caussade, 7.XI.2011, leg. T. Struyve (SDEI).

Paratypes: 1 o $^{\top}$ e 1 \% , stessa provenienza (IRSNB).

Descrizione: Lunghezza $0,9 \mathrm{~mm}$. Corpo lucido e nerobruno, base dell'addome e pigidio bruni, antenne brune con i tre antennomeri basali gialli, quarto a sesto giallo-bruni, zampe gialle. Occhi più lunghi della regione postoculare, in visione dorsale. Secondo antennomero più corto del primo, terzo più corto del secondo, quarto a decimo trasversi. Reticolazione di capo ed elitre superficiale, quella di pronoto e addome trasversa ed evanescente. Punteggiatura del capo fine ed evidente, quella del pronoto composta di alcuni punti. Granulosità delle elitre fitta, fine e saliente. Quinto urotergo libero del maschio con un granulo mediano saliente, situato più vicino alla base che al margine posteriore. Edeago fig. 75, spermateca fig. 76, sesto urotergo libero del maschio fig. 77.

Comparazioni: L'edeago della nuova specie ha struttura simile a quella di G. peruminima PACE, 2008 del Perù, ma questa ha edeago lungo $0,13 \mathrm{~mm}$ invece di 0,94 come in peruminima. L'estremità apicale dell'edeago di peruminima è larga, assai stretta nella nuova specie. Le due spine del margine posteriore del sesto urotergo libero del maschio di peruminima mancano nella nuova specie (fig. 77).
Etimologia: La nuova specie deriva il suo nome dalla Guyana Francese e dalla sua quasi microscopica taglia corporea di $0,9 \mathrm{~mm}$.

\section{Gyrophaena (Eumicrota) guyanensis n. sp}

(Figg. 20 e 78-80)

Materiale tipico: Holotype $\sigma^{*}$, Guyane Fr., Caussade, 7.XI.2011, leg. T. Struyve (IRSNB).

Paratypes: 5 esemplari, stessa provenienza (IRSNB, SDEI).

Descrizione: Lunghezza 1,4 mm. Corpo lucido e nero, base dell'addome bruna, antenne brune con i quattro antennomeri basali gialli, quinto giallo-bruno, zampe giallo-rossicce. Occhi più lunghi della regione postoculare, in visione dorsale. Secondo antennomero lungo quanto il primo, terzo più corto del secondo, quarto a decimo trasversi. Reticolazione di capo e pronoto superficiale, quella delle elitre trasversa e quasi forte, quella dell'addome poligonale irregolare evidente. Punteggiatura del capo molto sparsa ed evidente, quella del pronoto composta di otto punti molto distanziati. Granulosità delle elitre rada, fine e poco saliente, quella dell'addome riunita presso il margine posteriore. Quinto urotergo libero del maschio con due carene mediane lievemente convergenti all'indietro dove sta un tubercolo mediano molto saliente. Edeago fig. 78, spermateca fig. 79 , sesto urotergo libero del maschio fig. 80 .

Comparazioni: La struttura dell'edeago della nuova specie è simile a quella di G. plaumanni PACE, 2002 del Brasile. Ledeago della nuova specie è meno lungo, $0,2 \mathrm{~mm}$, di quello di plaumanni, $0,48 \mathrm{~mm}$, profondamente arcuato al lato ventrale, mentre quello di plaumanni non è arcuato. Tra le due spine marginali laterali del sesto urotergo libero del maschio di plaumanni vi sono tre piccoli lobi, nella nuova specie i medesimi sono due e appena sporgenti (fig. 80).

Etimologia: La nuova specie è dedicata alla Guyana Francese.

\section{Gyrophaena (Eumicrota) guytrina n. sp.}

(Figg. 21 e 81-83)

Materiale tipico: Holotype $\sigma^{\star}$, Guyane Fr., Barrage Petit Saut, 10.XI.2011, leg. T. Struyve (SDEI).

Paratype: 1 , stessa provenienza (IRSNB).

Descrizione: Lunghezza 1,5 mm. Corpo lucido e giallo-bruno, tre quarti posteriori delle elitre e uroterghi liberi quarto e quinto bruni, antenne bruno-rossicce con i tre antennomeri basali gialli, zampe gialle. Occhi 
più lunghi della regione postoculare, in visione dorsale. Secondo antennomero più corto del primo, terzo più corto del secondo, quarto a decimo trasversi. Reticolazione del capo molto superficiale, quella del pronoto trasversa ed evidente, quella delle elitre molto trasversa e forte, quella dell'addome evanescente. Punteggiatura del capo molto superficiale, quella del pronoto irregolarmente distribuita. Granulosità di elitre e addome fine e sparsa. Edeago fig. 81, spermateca fig. 82, sesto urotergo libero del maschio fig. 83 .

Comparazioni: L'edeago della nuova specie è simile a quello di G. atoma CASEY, 1906 del N America e Guyana Francese. Se ne distingue per l'ampia concavità ventrale dell'edeago, in atoma ad angolo acuto, per l'armatura genitale interna dell'edeago corta e non molto lunga come in atoma e per le due lunghe spine laterali del sesto urotergo libero del maschio, assenti in atoma.

Etimologia: La nuova specie prende nome dalle tre sporgenze del margine posteriore del sesto urotergo libero del maschio.

\section{Gyrophaena (Eumicrota) cayennensis n. sp. (Figg. 22 e 84-85)}

Materiale tipico: Holotype $\sigma^{\star}$, Guyane Fr., Barrage Petit Saut, 10.XI.2011, leg. T. Struyve (IRSNB).

Descrizione: Lunghezza 1,2 $\mathrm{mm}$. Corpo lucido e bruno, base delladdome rossiccia, antenne brune con i due antennomeri basali e l'undicesimo giallo-rossicci, zampe giallo-rossicce. Occhi più lunghi della regione postoculare, in visione dorsale. Secondo antennomero più corto del primo, terzo più corto del secondo, quarto a decimo trasversi. Reticolazione del capo superficiale, quella del pronoto quasi invisibile, quella delle elitre evidente, quella dell'addome assente tranne sul sesto urotergo libero del maschio dove è molto trasversa e molto superficiale. Granulosità dell'avancorpo poco fitta e saliente, quella dell'addome poco saliente e longitudinale. Edeago fig. 84, sesto urotergo libero del maschio fig. 85.

Comparazioni: Per la forma del sesto urotergo libero del maschio, la nuova specie è simile a G. bigranulata PACE, 2008 del Perù, ma le spine laterali del margine posteriore del sesto urotergo libero del maschio sono parallele nella nuova specie, convergenti all'indietro in bigranulata. La gibbosità ventrale dell'edeago della nuova specie è assente in bigranulata.

Etimologia: La nuova specie è dedicata alla capitale della Guyana Francese, Cayenne.
Gyrophaena (Eumicrota) exiliphallus n. sp.

(Figg. 23 e 86-87)

Materiale tipico: Holotype $\sigma^{\star}$, Guyane Fr., Barrage Petit Saut, 10.XI.2011, leg. T. Struyve (IRSNB).

Descrizione: Lunghezza 1,4 $\mathrm{mm}$. Corpo lucido e rossiccio, elitre e uroterghi liberi quarto e quinto bruni, antenne giallo-brune con i tre antennomeri basali gialli, zampe giallo-rossicce. Occhi più lunghi della regione postoculare, in visione dorsale. Secondo antennomero più corto del primo, terzo più corto del secondo, quarto a decimo trasversi. Reticolazione dell'avancorpo superficiale, quella dell'addome molto trasversa ed evanescente. Punteggiatura del capo invisibile, quella del pronoto sparsa. Granulosità delle elitre forte e saliente lungo la sutura, sul resto della superficie fine, invisibile ai lati esterni. Granulosità dell'addome presente solo lungo il margine posteriore di ciascun urotergo libero. Un forte punto mediano sulle elitre presso il margine posteriore. Un forte granulo sporgente dal profilo laterale delle elitre, situato presso l'angolo posteriore. Edeago fig. 86, sesto urotergo libero del maschio fig. 87.

Comparazioni: Per la forma dell'edeago e alcuni caratteri dell'habitus, come la presenza di forti granuli lungo la sutura delle elitre del maschio, la nuova specie è comparabile con G. guaranorum PACE, 2002 del Brasile. Se ne distingue per l'edeago lungo $0,14 \mathrm{~mm}$ invece di 0,25 come in guaranorum. La sinuosità ventrale dell'edeago della nuova specie presso la "crista apicalis" è stretta e non ampia come in guaranorum. La bozza alla base delle elitre del maschio e e'altra sul quinto urotergo libero del maschio di guaranorum sono assenti nella nuova specie. Il granulo laterale e sporgente delle elitre della nuova specie manca in guaranorum.

Etimologia: La nuova specie deriva il suo nome dall'edeago che ha forma esile.

Gyrophaena (Eumicrota) guyaspinosa n. sp.

(Figg. 24 e 88-89)

Materiale tipico: Holotype + , Guyane Fr., Caussade, 7.XI.2011, leg. T. Struyve (IRSNB).

Descrizione: Lunghezza 1,2 $\mathrm{mm}$. Corpo lucido e nerobruno, uroterghi liberi quarto e quinto neri, antenne brune con i tre antennomeri basali giallo pallido, zampe gialle. Occhi più lunghi della regione postoculare, in visione dorsale. Secondo antennomero più corto del primo, terzo più corto del secondo, quarto a decimo trasversi. Reticolazione del capo molto superficiale, quella del pronoto evanescente, quella delle elitre poligonale irregolare evidente, quella dell'addome forte. Punteggiatura del capo evidente e assente su una stretta fascia longitudinale mediana, quella del pronoto evidente e sparsa. Granulo- 
sità delle elitre poco fitta e saliente, quella dell'addome quasi invisibile. Spermateca fig. 88 , sesto urotergo libero della femmina fig. 89 .

Comparazioni: La nuova specie ha il sesto urotergo libero della femmina di forma molto caratteristica non comparabile con quella delle specie nordamericane e sudamericane note.

Etimologia: La nuova specie deriva il suo nome dalle due forti spine del margine posteriore del sesto urotergo libero della femmina e dalla Guyana Francese.

\section{Brachida paraensis PACE, 2002}

Brachida paraensis PACE, 2002: 133

1 ㅇ, Guyane Fr., Coralie, 15.XI.2011, leg. T. Struyve (IRSNB).

Distribuzione: Brasile.

\section{Brachida guyanensis n. sp.} (Figg. 25 e 90)

Materiale tipico: Holotype + , Guyane Fr., Caussade, 7.XI.2011, leg. T. Struyve (SDEI).

Paratype: $1 \uparrow$, stessa provenienza.

Descrizione: Lunghezza 2,4 mm. Corpo lucido e rossiccio, antenne brune con i quattro antennomeri basali gialli e undicesimo giallo-rossiccio, zampe giallo-rossicce. Occhi più corti della regione postoculare, in visione dorsale. Secondo antennomero lungo quanto il primo, terzo più corto del secondo, quarto a settimo più lunghi che larghi, ottavo a decimo lunghi quanto larghi. Reticolazione del corpo invisibile. Punteggiatura del capo fitta e molto superficiale, quella del pronoto non visibile, quella delle elitre fitta ed evanescente. Granulosità dell'addome fitta e molto superficiale. Spermateca fig. 90 .

Comparazioni: Per la forma della spermateca, la nuova specie è simile a B. aymaraorum PACE, 2002 della Bolivia. La nuova specie se ne distingue per gli occhi meno ridotti, per il quinto urotergo libero della femmina senza reticolazione e non con reticolazione evidente come in aymaraorum. Il bulbo distale della spermateca è sferico nella nuova specie, piriforme in aymaraorum. La parte intermedia della spermateca della nuova specie è corta, quella di aymaraorum lunga.

Etimologia: La nuova specie è dedicata alla Guyana Francese.

\section{Brachychara bartolozzii PACE, 1996}

Brachychara bartolozzii PACE, 1996: 413

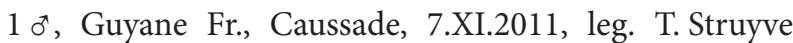
(SDEI).

Distribuzione: Ecuador.

Brachychara struyvei n. sp.

(Figg. 26 e 91-27)

Materiale tipico: Holotype $\sigma^{*}$, Guyane Fr., Coralie, 15.XI.2011, leg. T. Struyve (IRSNB).

Descrizione: Lunghezza 1,3 mm. Corpo lucido e giallo-rossiccio, antenne giallo-brune con i tre antennomeri basali giallo-rossicci, zampe giallo-rossicce. Occhi più lunghi della regione postoculare, in visione dorsale. Secondo antennomero più corto del primo, terzo più corto del secondo, quarto a decimo trasversi. Reticolazione del capo superficiale, quella del pronoto trasversa ed evanescente, quella delle elitre trasversa ed evidente, quella dell'addome poligonale irregolare superficiale. Punteggiatura di capo e pronoto molto evanescente. Granulosità di elitre e addome fine e fitta. Uroterghi liberi terzo e quarto del maschio con tre carene marginali posteriori a ciascun lato della linea mediana. Edeago fig. 91.

Comparazioni: L’edeago della nuova specie è simile a quello di B. catharinensis PACE, 2002 del Brasile. Se ne distingue per la parte apicale ventrale dell'edeago bisinuosa e non quasi rettilinea come in catharinensis. Larmatura genitale interna dell'edeago della nuova specie è corta, quella di catharinensis lunga.

Etimologia: La nuova specie è dedicata al suo raccoglitore Tim Struyve, entomologo e consulente di pianificazione di Mechelen, Belgio.

\section{PLACUSINI}

Placusa graciensis comb. n., nom. n.

(Figg. 92-94)

Amischa pumilio BeRnHAUER, 1927: 253

nec Placusa pumilio Gravenhorst, 1802: 98

Materiale esaminato: Holotype $\sigma^{*}$, Alta Gracia, Cord., XII.1920, ex nido Heromyrmex heyeri Fabr., Amischa pumilio Brnh. Typus unic., don Bruch (FMNHC).

Distribuzione: Cile. 

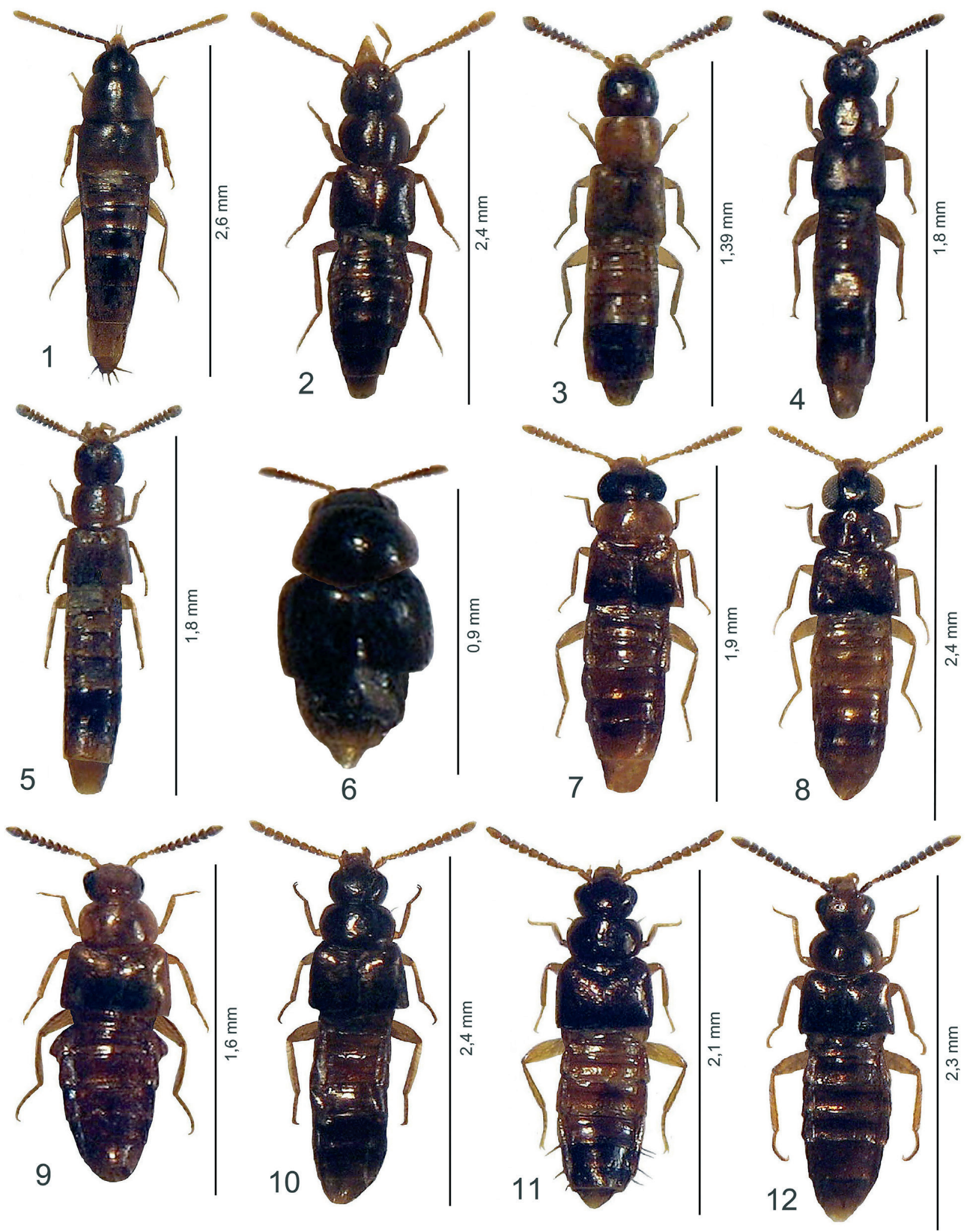

Figg. 1-12: Habitus. 1. Myllena guyanensis n. sp.; 2. Amazonopora sulcicollis n. sp.; 3. Hygronoma guyanensis n. sp.; 4. Hygronoma struyvei n. sp.; 5. Hygronoma filiformis n. sp.; 6. Holobus guyanensis n. sp.; 7. Gyrophaena (Phanerota) coraliensis n. sp.; 8. Gyrophaena (Phanerota) amazonica n. sp.; 9. Gyrophaena (Gyrophaena) comtensis n. sp.; 10. Gyrophaena (Gyrophaena) perstriata n. sp.; 11. Gyrophaena (Gyrophaena) ouaquiensis n. sp.; 12. Gyrophaena (Gyrophaena) pilositheca n. sp. 


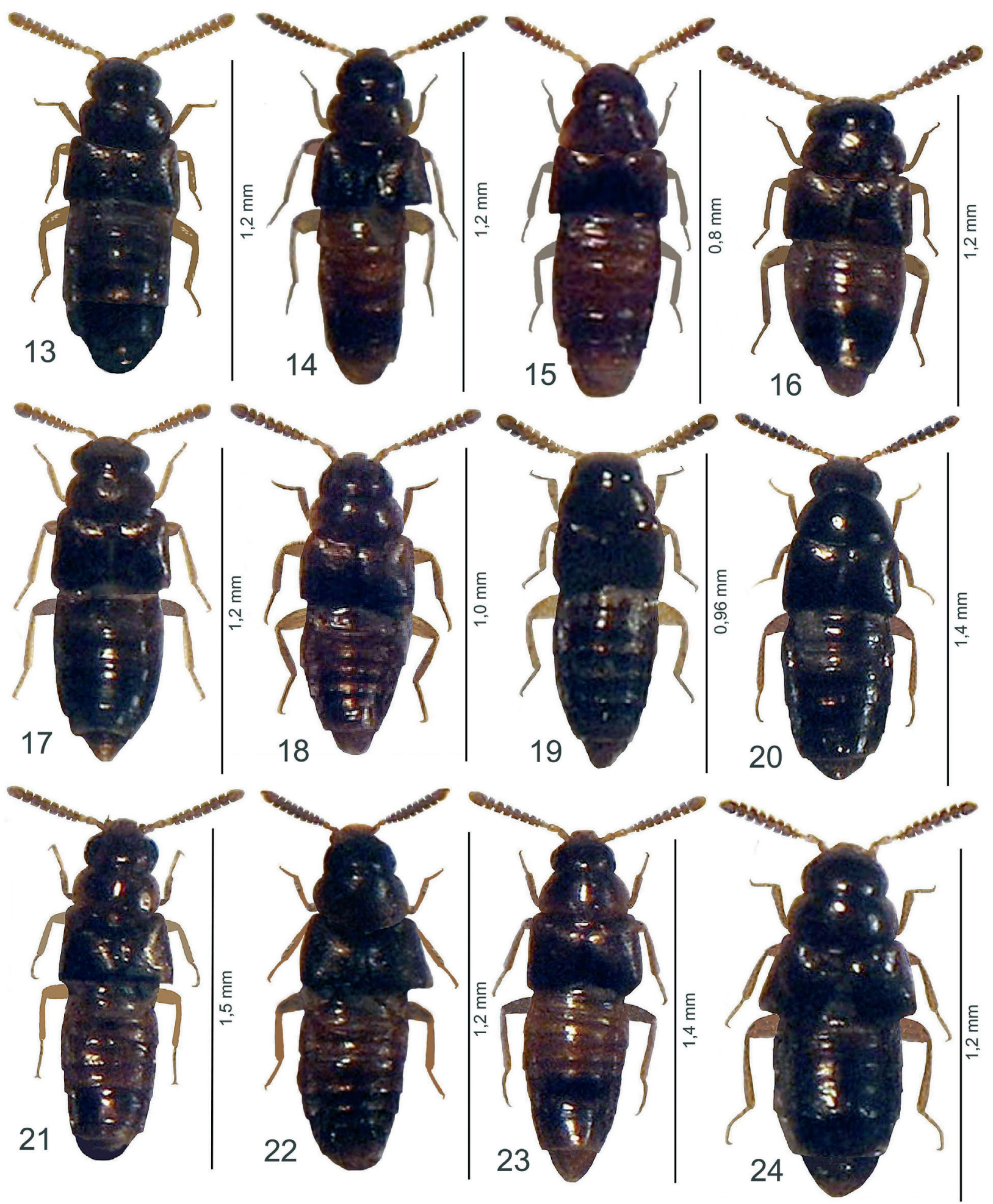

Figg. 13-24: Habitus. 13. Gyrophaena (Eumicrota) guyabicornis n. sp.; 14. Gyrophaena (Eumicrota) perminima n. sp.; 15. Gyrophaena (Eumicrota) persubtilis n. sp.; 16. Gyrophaena (Eumicrota) guyasimplex n. sp.; 17. Gyrophaena (Eumicrota) struyvei n. sp.; 18. Gyrophaena (Eumicrota) schoelcheri n. sp.; 19. Gyrophaena (Eumicrota) microguyana n. sp.; 20. Gyrophaena (Eumicrota) guyanensis n. sp.; 21. Gyrophaena (Eumicrota) guytrina n. sp.; 22. Gyrophaena (Eumicrota) cayennensis n. sp.; 23. Gyrophaena (Eumicrota) exiliphallus n. sp.; 24. Gyrophaena (Eumicrota) guyaspinosa n. sp. 


\section{Placusa amazonica n. sp.}

(Figg. 27 e 95-98)

Materiale tipico: Holotype $\sigma^{\star}$, Guyane Fr., Coralie, 15.XI.2011, leg. T. Struyve (SDEI).

Paratypes: 3 ㅇ 우 , stessa provenienza (IRSNB).

Descrizione: Lunghezza 1,6 mm. Corpo lucido e giallo-rossiccio, elitre e uroterghi liberi terzo a quinto bruni, antenne nere con i tre antennomeri basali gialli, quarto giallo-bruno, zampe giallo-rossicce. Occhi più corti della regione postoculare, in visione dorsale. Secondo antennomero più corto del primo, terzo più corto del secondo, quarto a decimo molto trasversi. Reticolazione del capo evidente sulla fronte, quella sul resto del capo e del corpo superficiale. Punteggiatura del corpo molto fitta e superficiale, assente sulla fronte. Edeago fig. 95, spermateca (bulbo distale della spermateca schiacciato non da me, ma da chi l'ha incollata sul cartellino porta insetti) fig. 96, sesto urotergo libero del maschio fig. 97 , sesto urotergo libero della femmina fig. 98.

Comparazioni: La nuova specie deriva il suo nome dall'Amazzonia.

Etimologia: L'edeago della nuova specie è simile a quello di P. vilis PACE, 1990 del Suriname. Questa specie ha lama sternale dell'edeago priva di dentini dorsali e ventrali e il flagello interno dell'edeago descrive tre spire, mentre quello della nuova specie una sola. Ledeago della nuova specie è lungo $0,21 \mathrm{~mm}$, quello di vilis $0,26 \mathrm{~mm}$. La spermateca della nuova specie ha bulbo distale globulare, quello di vilis è indistinto rispetto alla parte intermedia della stessa spermateca.

\section{Ringraziamenti}

Per avermi affidato in studio il materiale oggetto del presente lavoro rivolgo i miei più cordiali ringraziamenti al collega e naturalista belga Tim Struyve di Mechelen. Per il prestito di tipi ringrazio il Dr. Didier Drugmand de l'Institut royal des Sciences naturelles de Belgique, il Dr. Lothar Zerche dello SDEI di Müncheberg e il Dr. A. Newton del Field Museum of Natural History di Chicago.

\section{Bibliografia}

Ahn, K. J. \& Ashe J. S. 1999: Two new species of Amazonopora PACE 1996 (Coleoptera Staphylinidae Aleocharinae) from Peru and French Guiana with a discussion of its phylogenetic relationships. - Tropical Zoology 12: 125-136.

Bernhauer, M. 1908: Beitrag zur Staphylinidenfauna von Südamerika. - Archiv für Naturgeschichte 74: 283-372.

Bernhauer, M. 1927: Zur Staphylinidenfauna Südamerika, insbesondere Argentiniens. - Archiv für Naturgeschichte 91: 229-264.

Cameron, M. 1930: New species of Staphylinidae from the Belgian Congo. - Revue de Zoologie et Botanique africaines 19: 405-421.

Casey, T. L. 1906: Observations on the Staphylinid groups Aleocharinae and Xantholinini chiefly of America. Transactions of the Academy of Science of St. Louis 16: 125-435.

Castelnau (= LAPorte), F. L. N. 1835: Études entomologiques, ou description d'insectes nouveaux, et observations sur la synonymie. Première partie. Méquignon-Marvis Père et Fils: 159 pp.

Erichson, W. F. 1837: Die Käfer der Mark Brandenburg Vol. I, Abt. 1. 384 pp. - Berlin.

ERICHSON, W. F. 1840: Genera et Species Staphylinorum Insectorum Coleopterorum Familiae. - Berlin: $954 \mathrm{pp}$.

Frank, J. H. 1972: The genus Oligota Mannerheim in the Caribbean Region (Coleóptera: Staphylinidae). The Coleopterists Bulletin 26: 125-146.

Gravenhorst, J. L. C. 1802: Coleoptera Brunsvicensia: 206 pp. - Brunsvigae.

KLIMASZEwski, J. 1979: A revision of the Gymnusini and Deinopsini of the world. Coleoptera: Staphylinidae, Aleocharinae. - Agriculture Canada, Ottawa, Monograph $\mathrm{N}^{\circ}$ 25: 169 pp.

KraAtZ, G. 1857: Genera Aleocharinorum Illustrata. Linnaea Entomologica 11: 1-43.

KraAtz, G. 1859a: Die Staphyliniden-Fauna von Ostindien, insbesonders der Insel Ceylan. - Archiv für Naturgeschichte 25: 1-196.

KraAtZ G. 1859b: Zur kritischen Kenntniss der in Gay's Historia fisica y politica von Solier beschriebenen Staphylinen. - Berliner Entomologische Zeitschrift, 3: 1-16.

KraAtz, G. 1862: Synonymische Bemerkungen. Ueber Coleopteren. - Berliner entomologische Zeitschrift 6: 298-300. 
Mulsant, E. \& Rey, Cl. 1874: Tribu des Brévipennes: Famille des Aléochariens: Septième Branche: Myrmédoniaires. - Annales de la Société d'Agriculture de Lyon (4) 6 (1873): 33-738.

PACE, R. 1987: Aleocharinae dell'isola della Guadalupa (Antille) (Coleoptera: Staphylinidae). - Folia Entomologica Hungarica 48: 187-205.

PACE, R. 1990a: Nuovi Falagriini, Hoplandriini ed Aleocharini della Regione Neotropica (Coleoptera, Staphylinidae) (LXXX Contributo alla conoscenza delle Aleocharinae). - Giornale italiano d'Entomologia 5: $157-180$.

PACE, R. 1990b: Aleocharinae neotropiche del Museo Ungherese di Storia naturale (Coleoptera, Staphylinidae) (XC Contributo alla conoscenza delle Aleocharinae). - Annales Historico-Naturales Musei Nationalis Hungarici, Budapest, Pars Zoologica 81: 53-107, 264 figg.

PACE, R. 1991: Aleocharinae dell'Isola di S. Lucia (Antille) raccolte da E. Piva (Coleoptera, Staphylinidae). Bollettino del Museo civico di Storia Naturale di Verona 15: 151-162.

PACE, R. 1996a: Aleocharinae della Colombia e dell' Ecuador: Parte I (Coleóptera, Staphylinidae). - Revue Suisse de Zoologie 103: 395-437.

PACE, R. 1996b: Amazonopora manausensis, un notevole nuovo genere e nuova specie di Staphylinidae della tribù Diglottini (Coleoptera) $\left(130^{\circ}\right.$ Contributo alla conoscenza delle Aleocharinae). - Bollettino della Società entomologica italiana 128: 37-40, 5 figg.

PACE, R. 2002: Gyrophaenini e Bolitocharini del Sudamerica (Coleoptera, Staphylinidae). - Nouvelle Revue d'Entomologie (N.S.) 19: 115-139.
PACE, R. 2008: New records of Aleocharinae from Ecuador and Peru, with the description of new species, new subgenera and new genera (Coleoptera, Staphylinidae): pp. 225-398. - In: Giachino P. M. (ed.) 2008: Biodiversity of South America I. Memoirs on Biodiversity, World Biodiversity Association onlus, Verona, 1: $496 \mathrm{pp}$.

PACE, R. 2011: New and additional records, new genera and new species of Aleocharinae from Venezuela, Ecuador and Peru (Coleoptera, Staphylinidae). Bollettino del Museo Civico di Storia Naturale di Verona, Botanica Zoologia 35: 43-70.

SAY, T. 1834: Descriptions of new North American insects and observations on some already described. - Transactions of the American Philosophical Society 5: 409-470.

SeEvers, C. H. 1951: A Revision of the North American and European Staphylinid Beetles of the Subtribe Gyrophaenae (Aleocharinae, Bolitocharini). - Fieldiana Zoology 32: 660-762.

SHARP, D. 1876: Contribution to an insectfauna of the Amazon Valley, Staphylinidae. - Transactions of the Entomological Society of London 1: 27-424.

Sharp, D. S. 1883: Staphylindae. - Biologia CentraliAmericana, Insecta, Coleoptera, Vol. 1 (2): 145-312.

Smetana, A. 2004: Aleocharinae, pp. 353-494. - In: I. LöBl \& A. Smetana (editors). Catalogue of Palaearctic Coleoptera, Vol. 2. - Stenstrup: Apollo Books: $942 \mathrm{pp}$. 

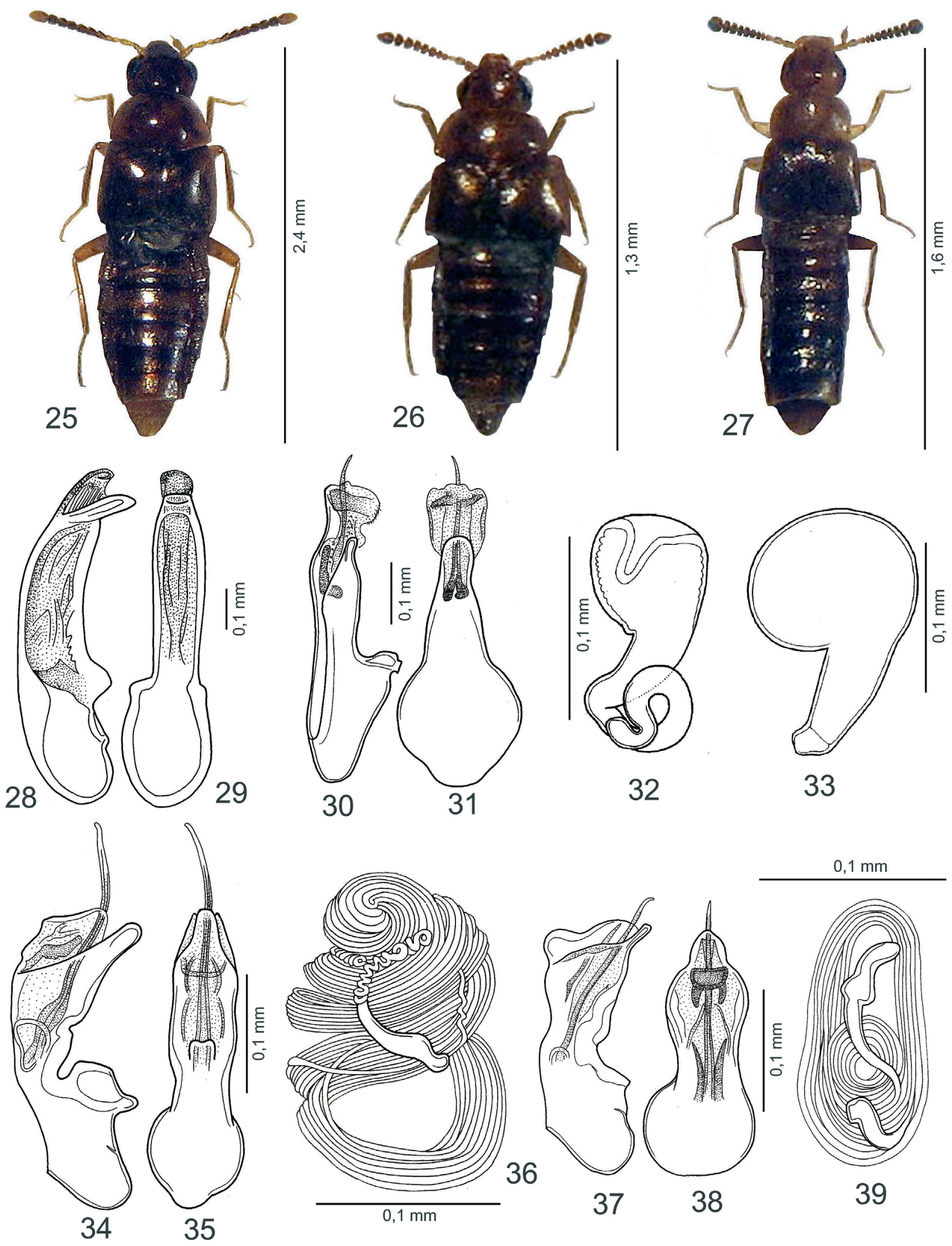

Figg. 25-39: Habitus, edeago in visione laterale e ventrale e spermateca. 25. Brachida guyanensis n. sp.; 26. Brachychara struyvei n. sp.; 27. Placusa amazonica n. sp.; 28-29. Metadeinopsis longicornis (Sharp, 1876); 30-32. Myllena guyanensis n. sp.; 33. Amazonopora sulcicollis n. sp.; 34-36. Hygronoma guyanensis n. sp.; 37-39. Hygronoma struyvei n. sp. 

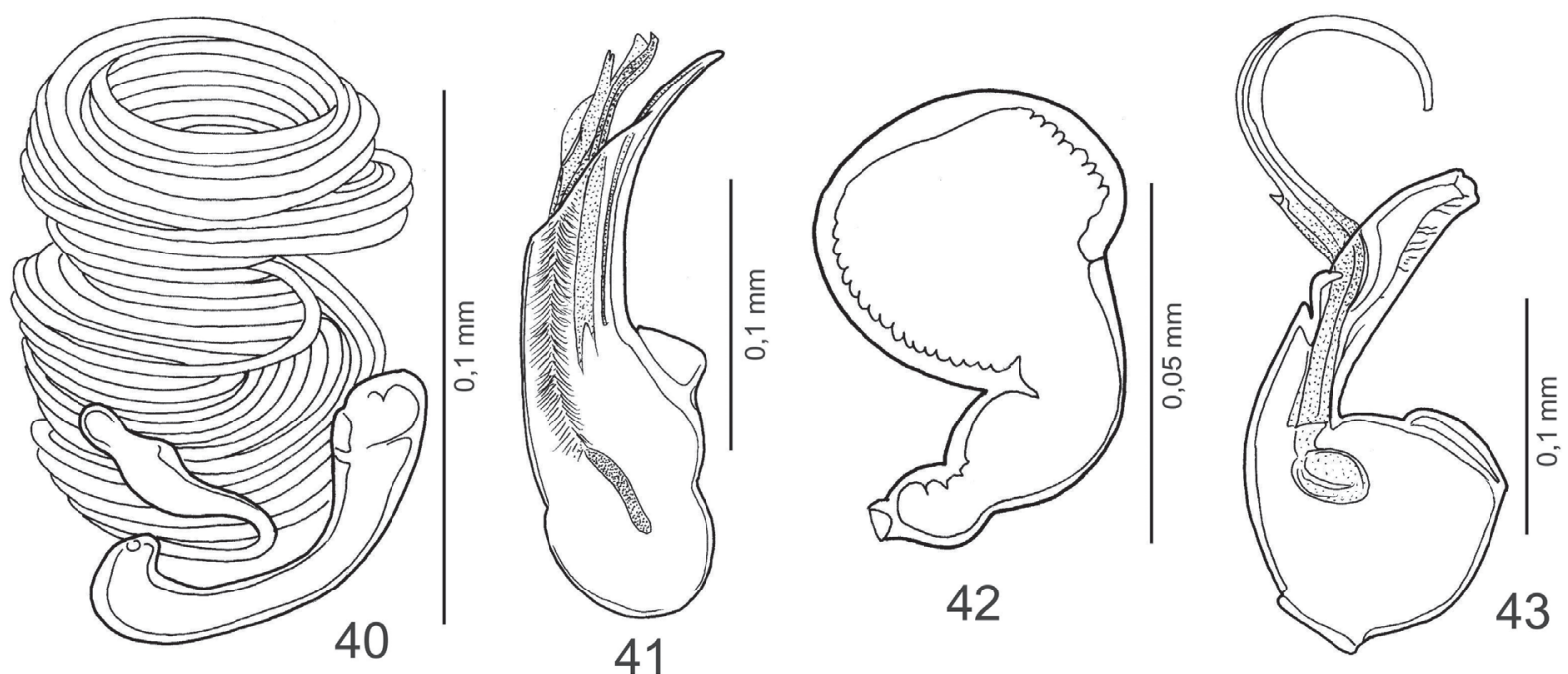

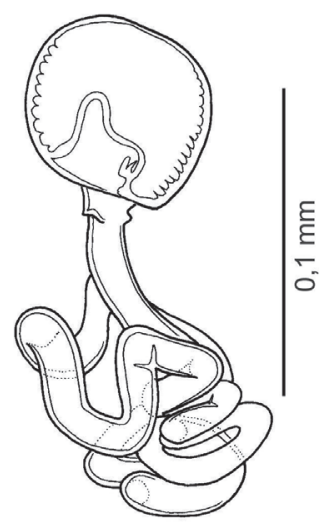

44

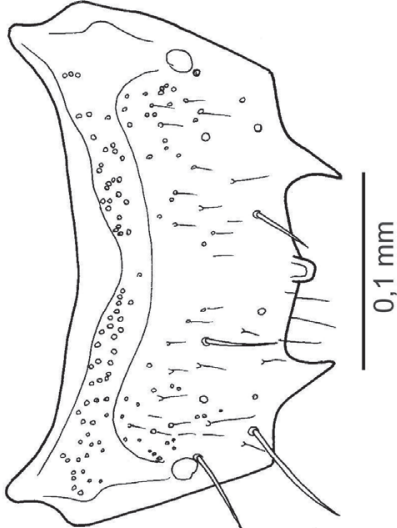

45
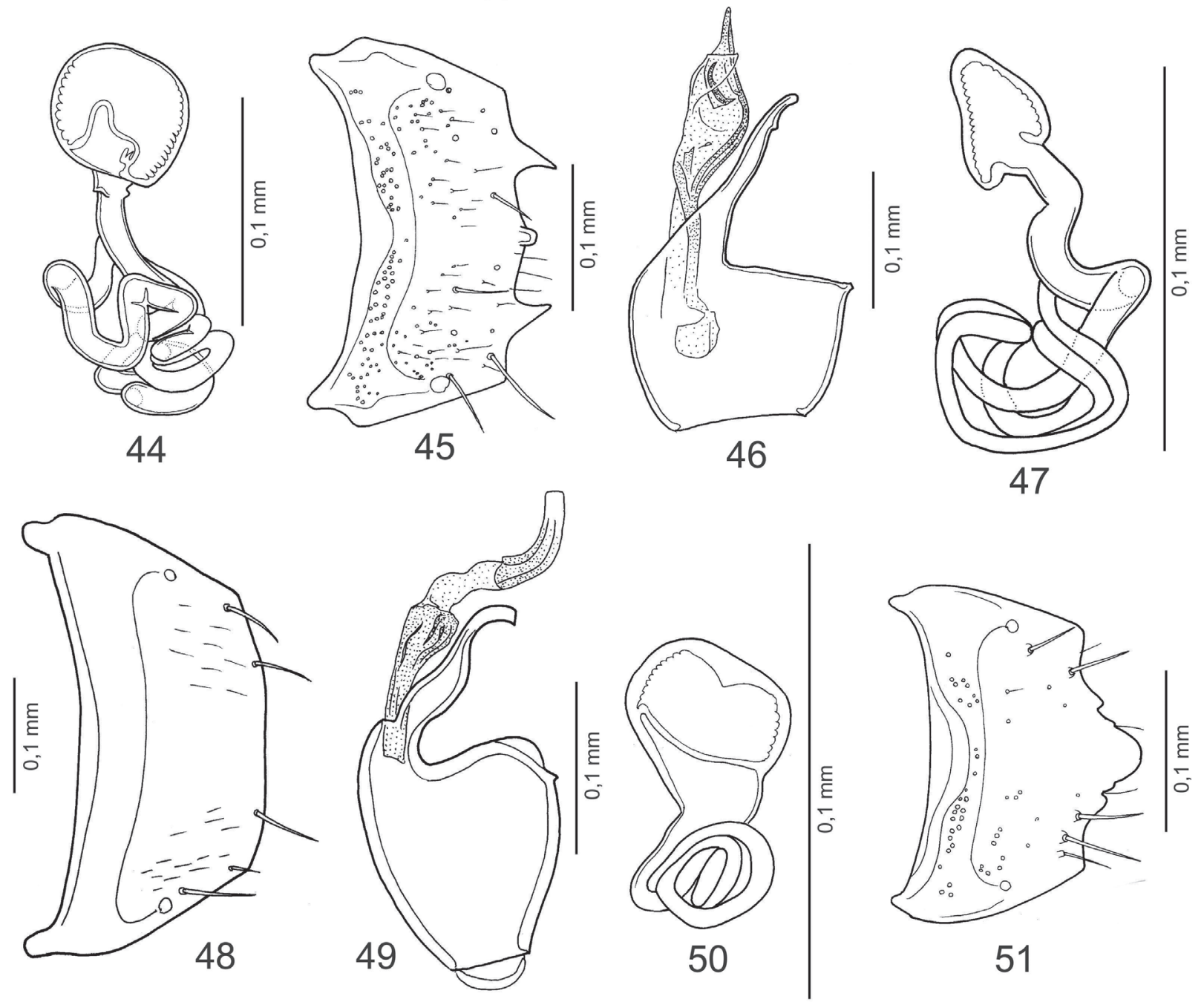

51

Figg. 40-51: Spermateca, edeago in visione laterale e sesto urotergo libero del maschio. 40. Hygronoma filiformis n. sp.; 41-42. Holobus guyanensis n. sp.; 43-45. Gyrophaena (Phanerota) coraliensis n. sp.; 46-48. Gyrophaena (Phanerota) amazonica n. sp.; 49-51. Gyrophaena (Gyrophaena) comtensis n. sp. 


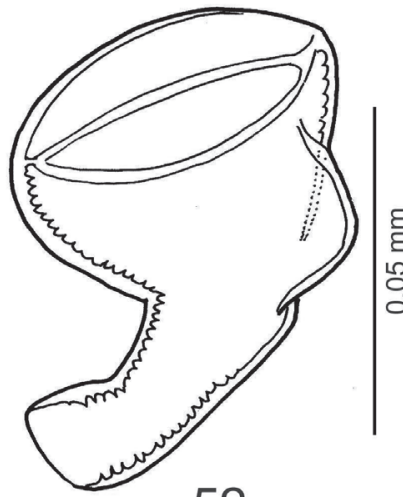

52

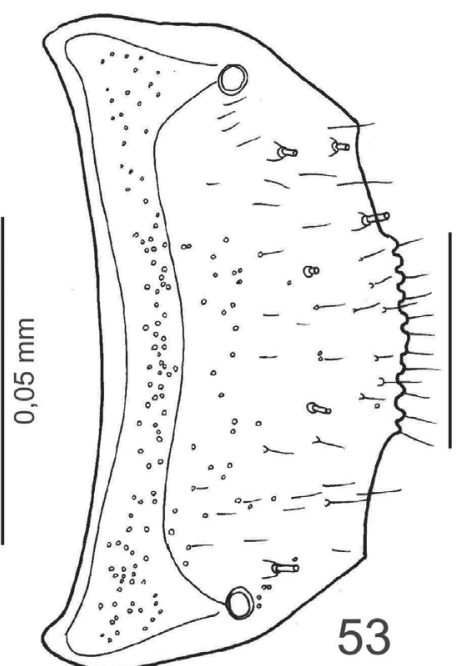

53

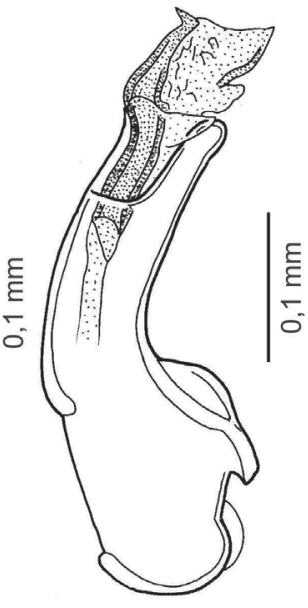

54
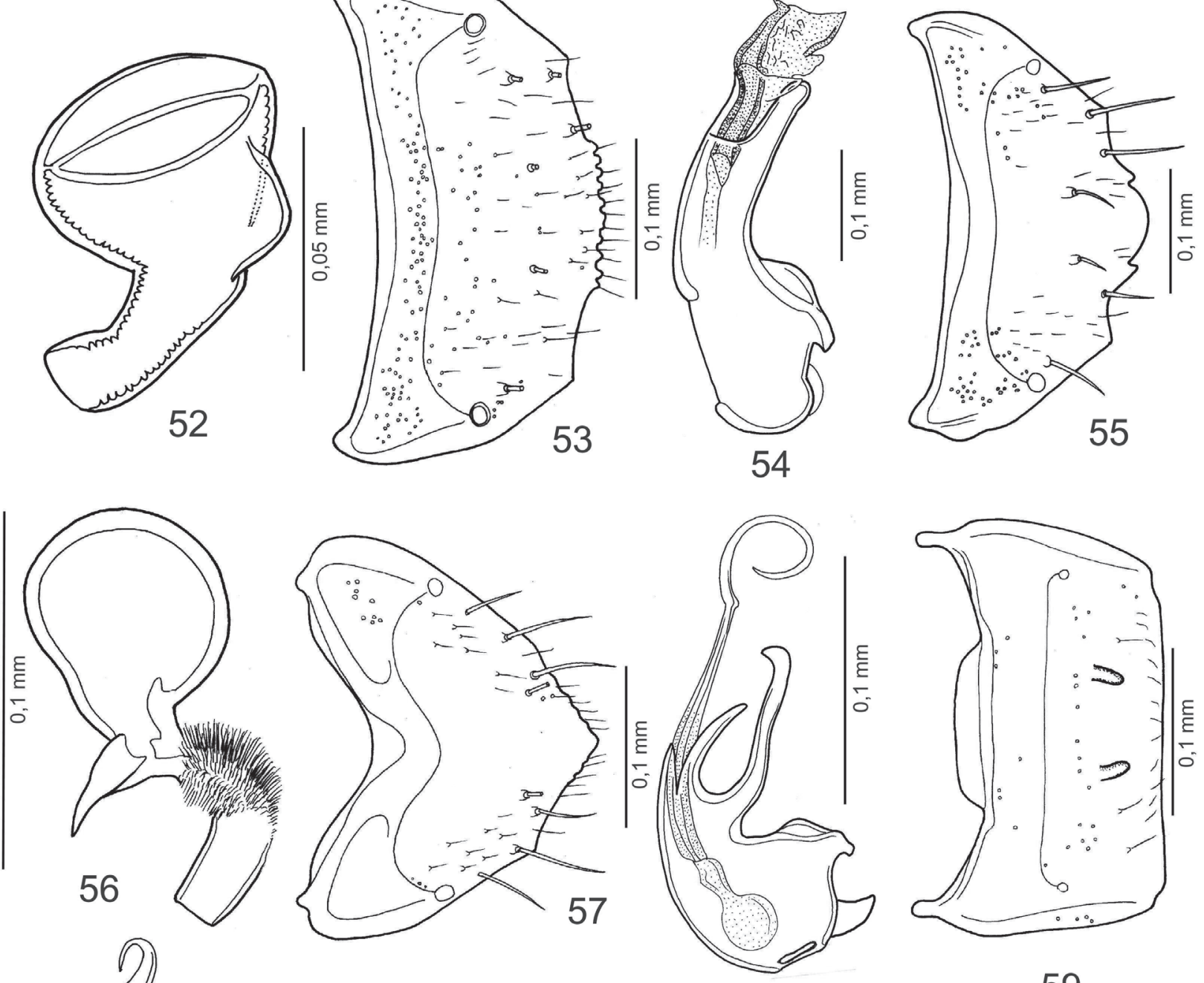

58

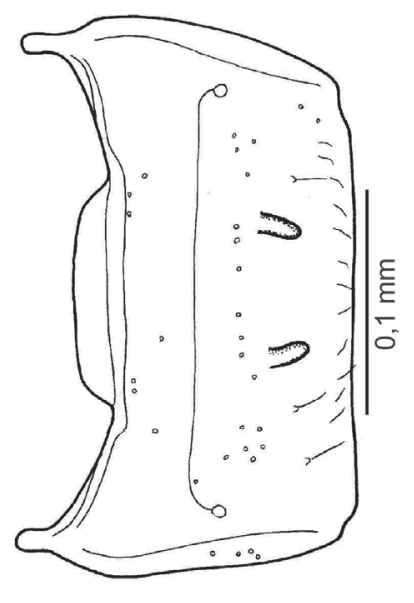

59
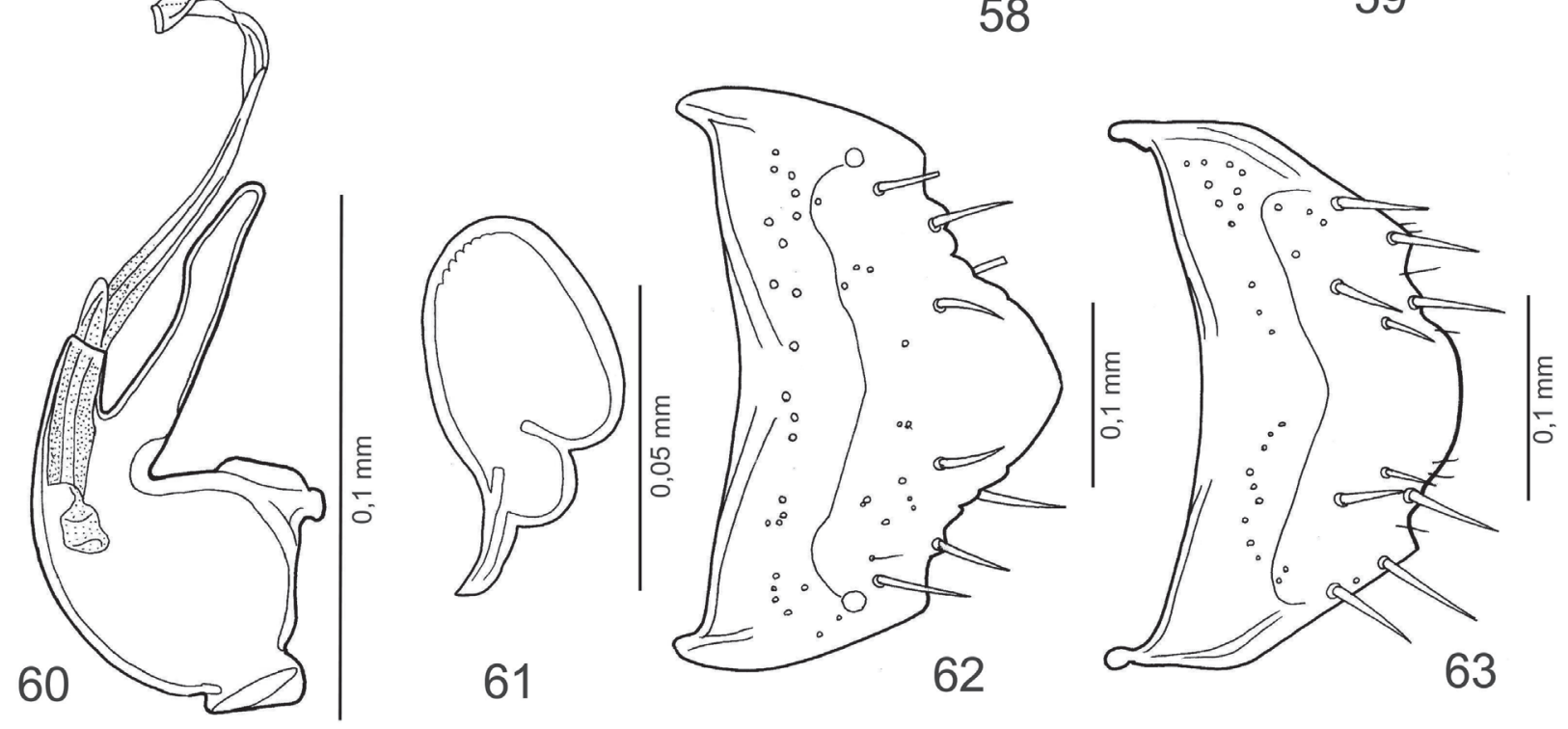

Figg. 52-63: Spermateca, sesto urotergo libero della femmina (53, 57 e 63) e del maschio $(55,59$ e 63) e edeago in visione laterale. 52-53. Gyrophaena (Gyrophaena) perstriata $\quad$ n. sp.; 54-55. Gyrophaena (Gyrophaena) ouaquiensis n. sp.; 56-57. Gyrophaena (Gyrophaena) pilositheca n. sp.; 58-59. Gyrophaena (Eumicrota) guyabicornis n. sp.; 60-63. Gyrophaena (Eumicrota) perminima n. sp. 


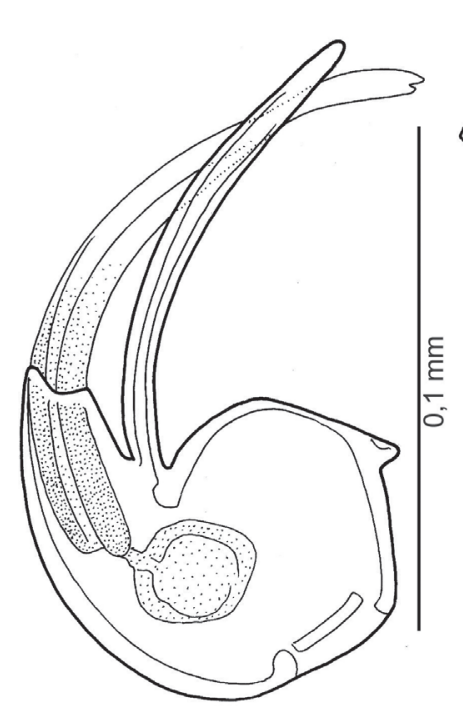

64
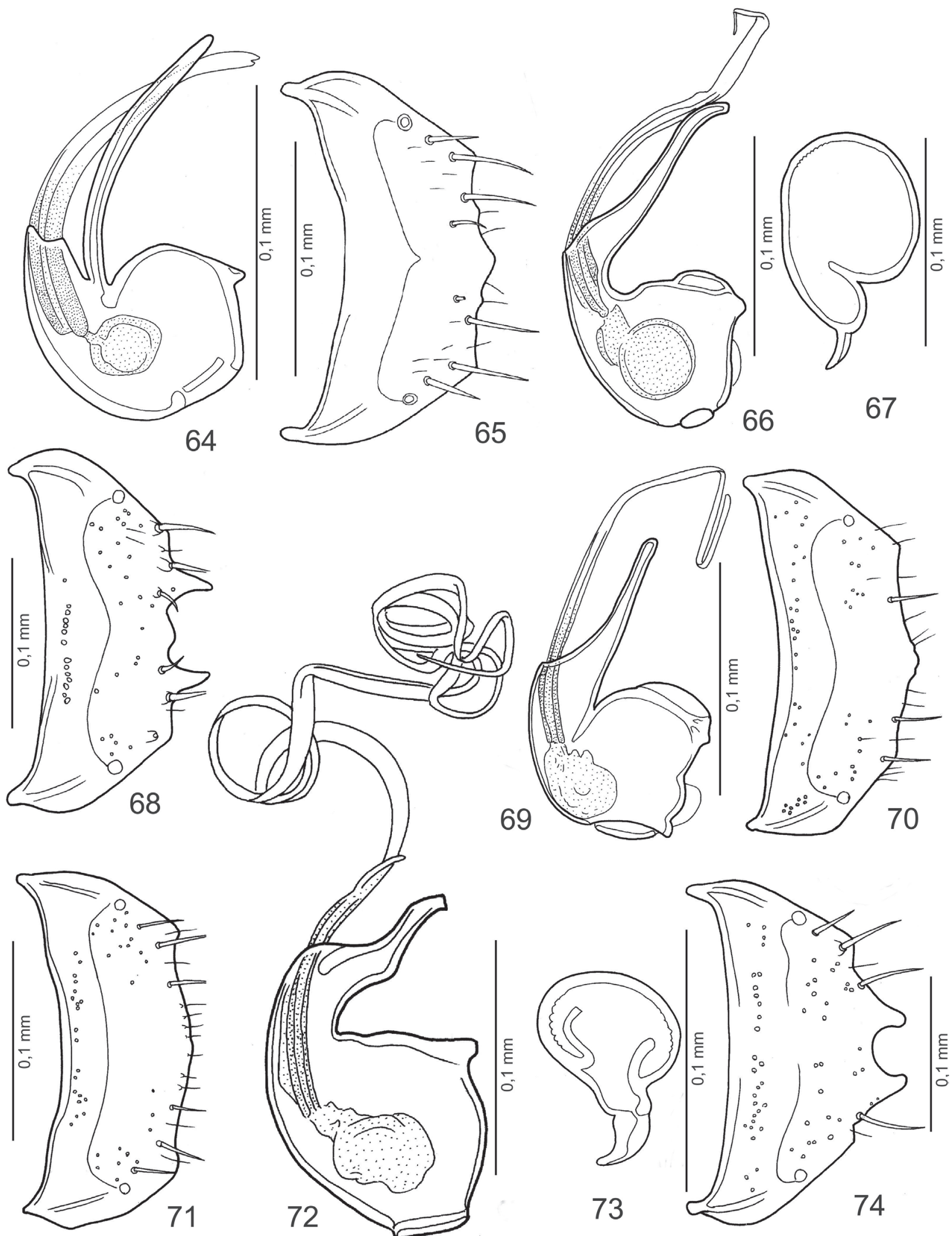

Figg. 64-74: Edeago in visione laterale, sesto urotergo libero del maschio e spermateca. 64-65. Gyrophaena (Eumicrota) persubtilis n. sp.; 66-68. Gyrophaena (Eumicrota) guyasimplex n. sp.; 69-71. Gyrophaena (Eumicrota) struyvei n. sp.; 72-74. Gyrophaena (Eumicrota) schoelcheri n. sp. 

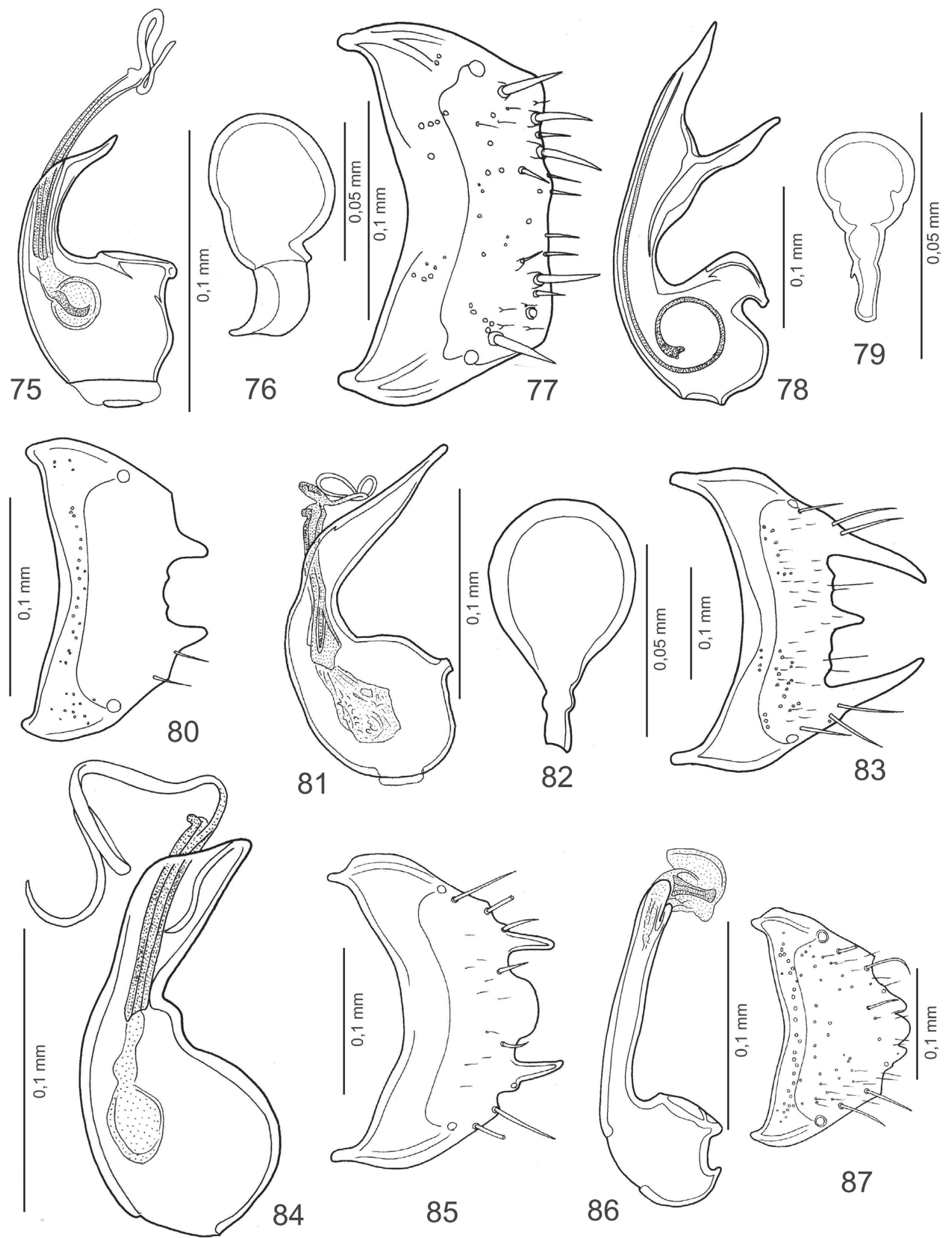

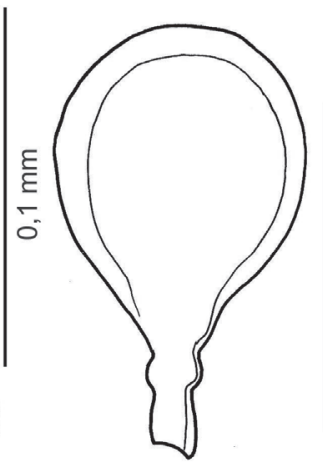

82
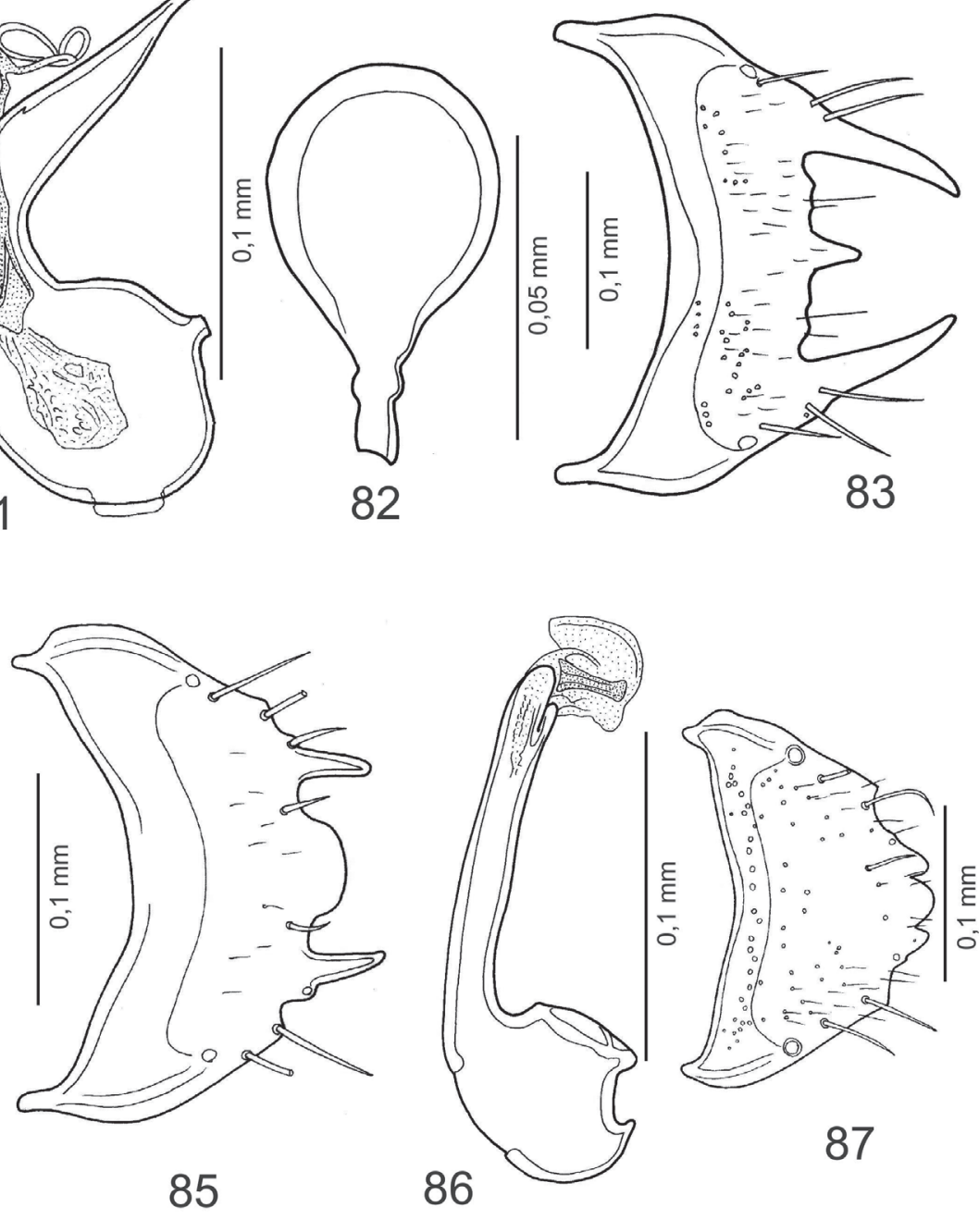

87

Figg. 75-87: Edeago in visione laterale, spermateca e sesto urotergo libero del maschio. 75-77. Gyrophaena (Eumicrota) microguyana n. sp.; 78-80. Gyrophaena (Eumicrota) guyanensis n. sp.; 81-83. Gyrophaena (Eumicrota) guytrina n. sp.; 84-85. Gyrophaena (Eumicrota) cayennensis n. sp.; 86-87. Gyrophaena (Eumicrota) exiliphallus $\mathrm{n}$. sp. 

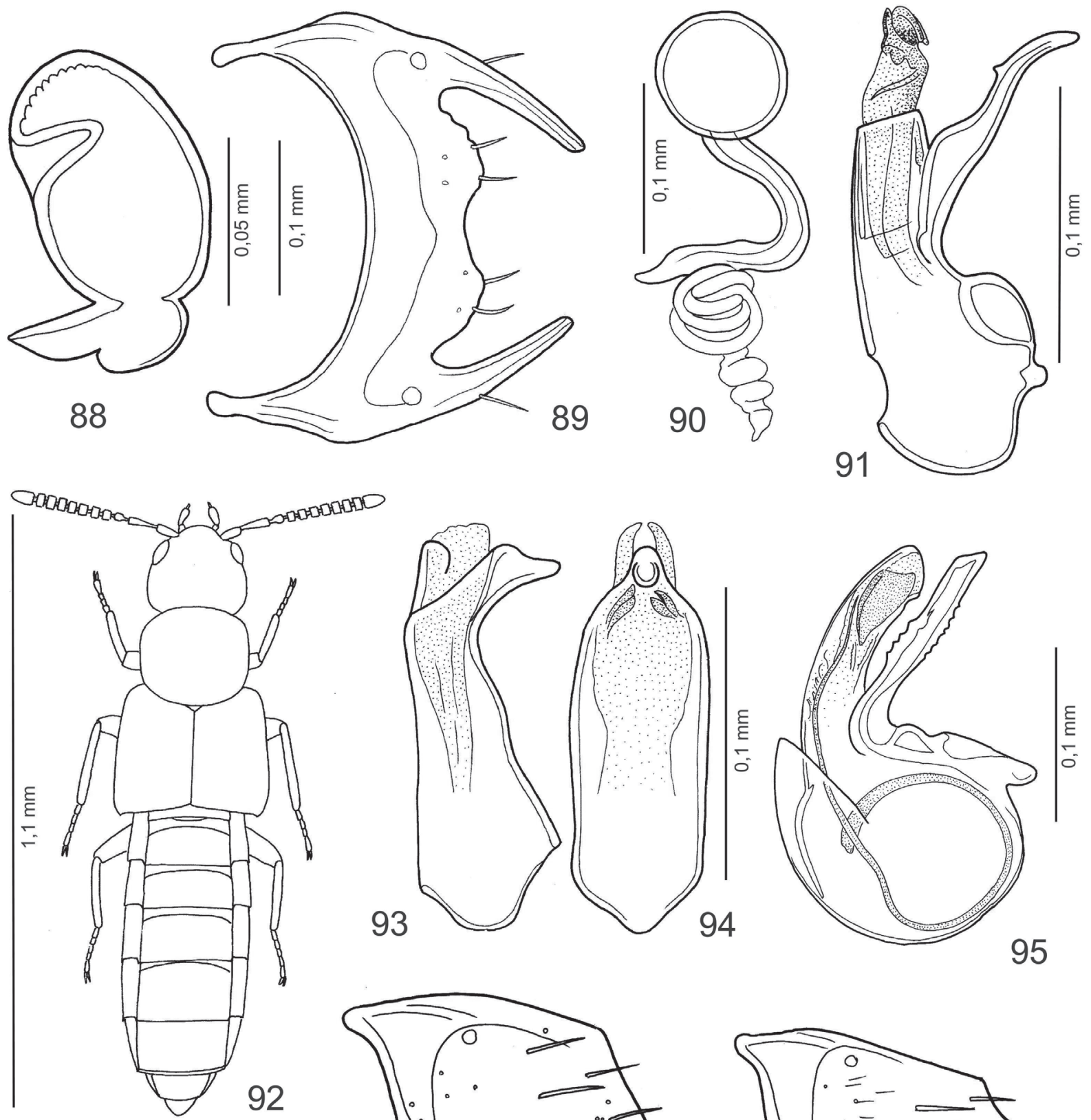

93
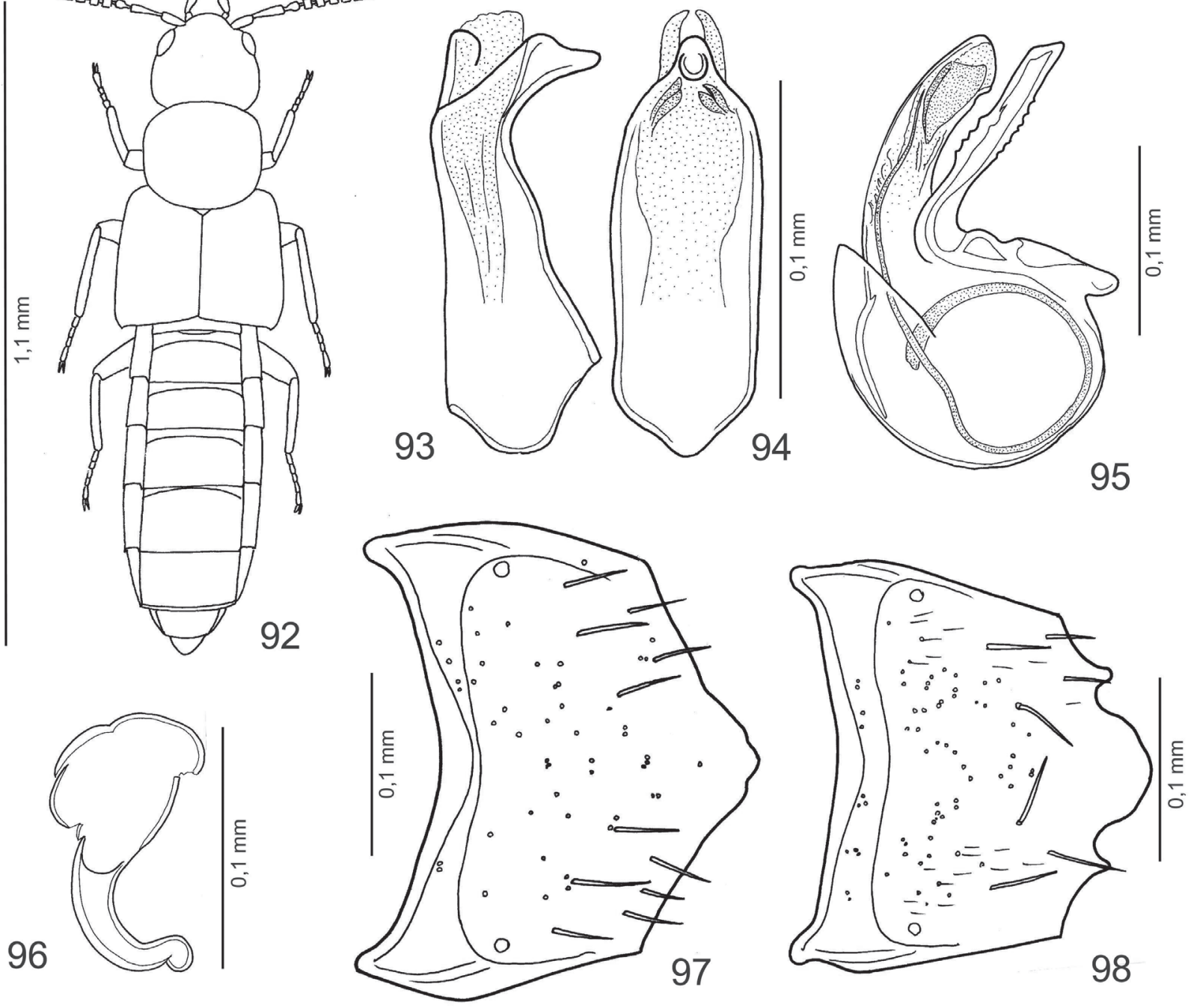

Figg. 88-98: Spermateca, sesto urotergo libero della femmina ( 89 e 98) e del maschio (97), edeago in visione laterale e ventrale e habitus. 88-89. Gyrophaena (Eumicrota) guyaspinosa n. sp.; 90. Brachida guyanensis n. sp.; 91. Brachychara struyvei n. sp.; 92-94. Placusa graciensis (Bernhauer) nom. nov., comb. n., Holotype; 95-98. Placusa amazonica n. sp. 